\title{
Origins of Selectivity in Pericyclic Reaction Cascades for the Synthesis of Gambogin and Lateriflorone
}

Amy E. Hayden, Hao Xu, K.C. Nicolaou, and K.N. Houk

\section{Supporting Information}

All ground and transition state structures were fully optimized at the B3LYP/6-31G(d) level of theory, with Gaussian 03. ${ }^{1}$ All structures were validated as minima or firstdegree saddle points using frequency analysis.

\footnotetext{
${ }^{1}$ Gaussian 03, revision C.02; Frisch, M.J.; Trucks, G.W.; Schlegel, H.B.; Scuseria, G.E.; Robb, M.A.; Cheeseman, J.R.; Montgomery, J.A., Jr.; Vreven, T.; Kudin, K.; Burant, J.C.; Millam, J.M.; Iyengay, S.S.; Tomasi, J.; Barone, V.; Mennucci, B.; Cossi, M.; Scalmani, G.; Rega, N.; Petersson, G.A.; Nakatsuji, H.; Hada, M.; Ehara, M.; Toyota, K.; Fukuda, R.; Hasegawa, J.; Ishida, M.; Nakajima, T.; Honda, Y.; Kitao, O.; Nakai, H.; Klene, M.; Li, X.; Knox, J.E.; Hratchian, H.P.; Cross, J.B.; Adamo, C.; Jaramillo, J.;

Comperts, R.; Startmann, R.E.; Yazyev, O.; Austin, A.J.; Cammi, R.; Pomelli, C.; Ochterski, J.W.; Ayala, P.Y.; Morokuma, K.; Voth, G.A.; Salvador, P.; Dannenbuerg, J.J.; Zakrzewski, V.G.; Dapprich, S.;

Daniels, A.D.; Strain, M.C.; Farkas, O.; Malick, D.K.; Rabuck, A.D.; Raghavachari, K.; Foresman, J.B.; Ortiz, J.V.; Cui, Q.; Baboul, A.G.; Clifford, S.; Cioslowski, J.; Stefanov, B.B.; Liu, G.; Liashenko, A.; Piskorz, P.; Komaromi, I.; Martin, R.L.; Fox, D.J.; Keith, T.; Al-Laham, M.A.; Peng, C.Y.; Nanayakkara, A.; Challacombe, M.; Gill, P.M.W.; Johnson, B.; Chem, W.; Wong, M.W., Gonzalez, C.; Pople, J.A.; Gaussian, Inc.: Wallingford CT, 2004.
} 
Minima and transition structure geometries and energies. Energies are in Hartrees, distances and coordinates in units of Angstroms.

Model System

3

\begin{tabular}{|c|c|c|c|}
\hline $\mathrm{C}$ & -0.025476 & -0.062202 & -0.274001 \\
\hline $\mathrm{C}$ & -0.190364 & -0.127908 & 1.110337 \\
\hline $\mathrm{C}$ & 0.926406 & -0.192322 & 1.943479 \\
\hline $\mathrm{C}$ & 2.206493 & -0.193767 & 1.388208 \\
\hline $\mathrm{C}$ & 2.386014 & -0.139589 & 0.001341 \\
\hline $\mathrm{C}$ & 1.254492 & -0.062956 & -0.838388 \\
\hline 0 & 1.453982 & -0.053687 & -2.200562 \\
\hline $\mathrm{C}$ & 1.182730 & 1.142419 & -2.986837 \\
\hline $\mathrm{C}$ & 2.257700 & 1.119726 & -4.086804 \\
\hline $\mathrm{C}$ & 1.313476 & 2.425109 & -2.152807 \\
\hline $\mathrm{C}$ & -0.187748 & 1.051914 & -3.635523 \\
\hline C & -0.949167 & -0.038881 & -3.706933 \\
\hline 0 & 3.643198 & -0.038036 & -0.544967 \\
\hline $\mathrm{C}$ & 4.446213 & -1.244614 & -0.793908 \\
\hline $\mathrm{C}$ & 3.593138 & -2.360789 & -1.418105 \\
\hline $\mathrm{C}$ & 5.082170 & -1.715731 & 0.516979 \\
\hline $\mathrm{C}$ & 5.456028 & -0.761447 & -1.814086 \\
\hline $\mathrm{C}$ & 6.780645 & -0.890743 & -1.760233 \\
\hline $\mathrm{H}$ & 2.086580 & 1.919287 & -4.816025 \\
\hline $\mathrm{H}$ & 3.249461 & 1.254601 & -3.643077 \\
\hline $\mathrm{H}$ & 2.229270 & 0.161235 & -4.613202 \\
\hline $\mathrm{H}$ & 0.542161 & 2.493185 & -1.381107 \\
\hline $\mathrm{H}$ & 2.295676 & 2.461818 & -1.671374 \\
\hline $\mathrm{H}$ & 1.216389 & 3.297339 & -2.808368 \\
\hline $\mathrm{H}$ & -0.519152 & 1.973712 & -4.115682 \\
\hline $\mathrm{H}$ & -1.904189 & -0.029268 & -4.225017 \\
\hline $\mathrm{H}$ & -0.637883 & -0.974803 & -3.252631 \\
\hline $\mathrm{H}$ & 4.232253 & -3.215456 & -1.662762 \\
\hline $\mathrm{H}$ & 2.820303 & -2.699577 & -0.719896 \\
\hline $\mathrm{H}$ & 3.102466 & -2.011106 & -2.329447 \\
\hline $\mathrm{H}$ & 5.661394 & -0.909567 & 0.977201 \\
\hline $\mathrm{H}$ & 4.309029 & -2.039601 & 1.219536 \\
\hline $\mathrm{H}$ & 5.745989 & -2.568981 & 0.341260 \\
\hline $\mathrm{H}$ & 4.996208 & -0.280124 & -2.676205 \\
\hline $\mathrm{H}$ & 7.411563 & -0.527015 & -2.566781 \\
\hline $\mathrm{H}$ & 7.293329 & -1.357599 & -0.923941 \\
\hline $\mathrm{H}$ & -1.191380 & -0.124968 & 1.533033 \\
\hline $\mathrm{H}$ & 0.806136 & -0.231505 & 3.022631 \\
\hline $\mathrm{H}$ & 3.085697 & -0.204404 & 2.023984 \\
\hline $\mathrm{H}$ & -0.885507 & -0.004553 & -0.932092 \\
\hline
\end{tabular}


Sum of Energies $=-773.332269$

4

\begin{tabular}{|c|c|c|c|}
\hline $\mathrm{C}$ & 0.094390 & -0.085260 & -0.033249 \\
\hline C & 0.126277 & -0.133018 & 1.316726 \\
\hline $\mathrm{C}$ & 1.379871 & -0.127181 & 2.057060 \\
\hline C & 2.558461 & -0.062278 & 1.417974 \\
\hline C & 2.698516 & -0.025947 & -0.087346 \\
\hline C & 1.324500 & -0.060596 & -0.832322 \\
\hline $\mathrm{O}$ & 1.278778 & -0.163177 & -2.053286 \\
\hline $\mathrm{O}$ & 3.507696 & 1.155678 & -0.281665 \\
\hline C & 3.506003 & 2.038742 & -1.430673 \\
\hline C & 3.878339 & 1.333331 & -2.745735 \\
\hline C & 4.623053 & 3.037334 & -1.063920 \\
\hline C & 2.197541 & 2.806444 & -1.551160 \\
\hline C & 1.412517 & 3.141286 & -0.527355 \\
\hline C & 3.467554 & -1.302316 & -0.567171 \\
\hline $\mathrm{C}$ & 4.857238 & -1.419667 & -0.007773 \\
\hline $\mathrm{C}$ & 5.422644 & -2.476078 & 0.598718 \\
\hline C & 4.741855 & -3.797346 & 0.861325 \\
\hline C & 6.857849 & -2.412619 & 1.067009 \\
\hline $\mathrm{H}$ & 4.012172 & 2.082393 & -3.535488 \\
\hline $\mathrm{H}$ & 4.827132 & 0.799368 & -2.627213 \\
\hline $\mathrm{H}$ & 3.103805 & 0.633546 & -3.059430 \\
\hline $\mathrm{H}$ & 4.407640 & 3.504009 & -0.098925 \\
\hline $\mathrm{H}$ & 5.587655 & 2.521472 & -1.002310 \\
\hline $\mathrm{H}$ & 4.695004 & 3.824063 & -1.822312 \\
\hline $\mathrm{H}$ & 1.935916 & 3.127800 & -2.559001 \\
\hline $\mathrm{H}$ & 0.506669 & 3.722881 & -0.674101 \\
\hline $\mathrm{H}$ & 1.642523 & 2.835464 & 0.489782 \\
\hline $\mathrm{H}$ & 2.854421 & -2.176675 & -0.322544 \\
\hline $\mathrm{H}$ & 3.495947 & -1.245187 & -1.662151 \\
\hline $\mathrm{H}$ & 5.465998 & -0.525880 & -0.134973 \\
\hline $\mathrm{H}$ & 5.268366 & -4.612498 & 0.345245 \\
\hline $\mathrm{H}$ & 4.770671 & -4.041094 & 1.932472 \\
\hline $\mathrm{H}$ & 3.697986 & -3.822117 & 0.540276 \\
\hline $\mathrm{H}$ & 7.470091 & -3.180184 & 0.572427 \\
\hline $\mathrm{H}$ & 7.310339 & -1.436744 & 0.865324 \\
\hline $\mathrm{H}$ & 6.931954 & -2.607050 & 2.146484 \\
\hline $\mathrm{H}$ & -0.804086 & -0.169944 & 1.879675 \\
\hline $\mathrm{H}$ & 1.340598 & -0.155130 & 3.142757 \\
\hline $\mathrm{H}$ & 3.496272 & -0.024172 & 1.964111 \\
\hline $\mathrm{H}$ & -0.834785 & -0.087006 & -0.594839 \\
\hline
\end{tabular}

Sum of Energies $=-773.308745$ 
4TS

$\begin{array}{lrrr}\mathrm{C} & 0.976341 & 1.997572 & 1.121845 \\ \mathrm{C} & 0.531797 & 3.142743 & 0.508555 \\ \mathrm{C} & -0.360405 & 3.079219 & -0.595425 \\ \mathrm{C} & -0.802862 & 1.858462 & -1.048780 \\ \mathrm{C} & -0.338206 & 0.640881 & -0.468154 \\ \mathrm{C} & 0.528432 & 0.681808 & 0.716524 \\ \mathrm{O} & 0.940254 & -0.370070 & 1.279335 \\ \mathrm{C} & 2.716961 & -1.145072 & -0.140316 \\ \mathrm{C} & 2.893972 & -2.514254 & 0.448758 \\ \mathrm{C} & 3.646209 & -0.089436 & 0.385990 \\ \mathrm{C} & 1.907695 & -0.968412 & -1.251710 \\ \mathrm{C} & 1.497406 & 0.267192 & -1.757625 \\ \mathrm{O} & -0.934437 & -0.549919 & -0.833843 \\ \mathrm{C} & -2.207101 & -0.960727 & -0.222483 \\ \mathrm{C} & -2.281372 & -2.450410 & -0.588612 \\ \mathrm{C} & -3.364862 & -0.193348 & -0.869236 \\ \mathrm{C} & -2.143285 & -0.821639 & 1.283493 \\ \mathrm{C} & -3.062667 & -0.256216 & 2.066153 \\ \mathrm{H} & 3.941472 & -2.841029 & 0.379072 \\ \mathrm{H} & 2.632807 & -2.487059 & 1.512825 \\ \mathrm{H} & 2.262438 & -3.259255 & -0.043041 \\ \mathrm{H} & 3.283717 & 0.925797 & 0.209969 \\ \mathrm{H} & 3.800885 & -0.215240 & 1.461486 \\ \mathrm{H} & 4.626643 & -0.184527 & -0.104422 \\ \mathrm{H} & 1.375185 & -1.843229 & -1.615825 \\ \mathrm{H} & 0.886707 & 0.287802 & -2.652939 \\ \mathrm{H} & 2.027460 & 1.186762 & -1.537001 \\ \mathrm{H} & -3.218690 & -2.886759 & -0.229394 \\ \mathrm{H} & -2.226475 & -2.571615 & -1.676047 \\ \mathrm{H} & -1.444703 & -2.992430 & -0.137782 \\ \mathrm{H} & -3.338201 & 0.869272 & -0.612355 \\ \mathrm{H} & -3.307847 & -0.292932 & -1.957902 \\ \mathrm{H} & -4.327403 & -0.598884 & -0.539988 \\ \mathrm{H} & -1.246541 & -1.252900 & 1.721494 \\ \mathrm{H} & -2.938809 & -0.235020 & 3.145672 \\ \mathrm{H} & -3.967424 & 0.204964 & 1.679097 \\ \mathrm{H} & -6997668 & 1.789480 & -1.889062 \\ \mathrm{H} & & 3.995210 & -1.071042 \\ \mathrm{H} & -1.63208 & 2.037001 & 1.987340\end{array}$

Sum of Energies $=-773.286902$ 


\begin{tabular}{|c|c|c|c|}
\hline C & 2.250410 & -1.637657 & -0.038758 \\
\hline $\mathrm{C}$ & 1.394073 & -2.385325 & -1.039515 \\
\hline C & 0.408450 & -1.651123 & -1.571547 \\
\hline $\mathrm{C}$ & 0.284627 & -0.249714 & -1.036892 \\
\hline $\mathrm{C}$ & 1.612277 & 0.580143 & -1.122595 \\
\hline $\mathrm{C}$ & 2.788703 & -0.363765 & -0.762007 \\
\hline $\mathrm{C}$ & 1.258936 & -1.212704 & 1.054875 \\
\hline C & 0.151638 & -0.262616 & 0.508088 \\
\hline $\mathrm{O}$ & 1.318953 & -1.568077 & 2.212259 \\
\hline C & -1.221647 & -0.626884 & 1.083969 \\
\hline $\mathrm{C}$ & -2.312582 & 0.328229 & 0.686396 \\
\hline C & -3.473522 & 0.056910 & 0.068092 \\
\hline C & -3.926556 & -1.314938 & -0.370568 \\
\hline $\mathrm{C}$ & -4.463551 & 1.160533 & -0.225084 \\
\hline $\mathrm{O}$ & 0.509229 & 1.065375 & 0.928372 \\
\hline $\mathrm{C}$ & 1.322892 & 1.711514 & -0.089225 \\
\hline $\mathrm{C}$ & 2.540851 & 2.317734 & 0.615280 \\
\hline $\mathrm{C}$ & 0.495086 & 2.840377 & -0.724917 \\
\hline $\mathrm{H}$ & 3.294850 & -0.689620 & -1.676305 \\
\hline $\mathrm{H}$ & -1.447278 & -1.657444 & 0.791727 \\
\hline $\mathrm{H}$ & -1.103533 & -0.634636 & 2.175836 \\
\hline $\mathrm{H}$ & -2.114490 & 1.364555 & 0.956158 \\
\hline $\mathrm{H}$ & -3.191150 & -2.099116 & -0.176564 \\
\hline $\mathrm{H}$ & -4.857394 & -1.596540 & 0.141505 \\
\hline $\mathrm{H}$ & -4.152940 & -1.323144 & -1.446066 \\
\hline $\mathrm{H}$ & -4.094707 & 2.137596 & 0.102338 \\
\hline $\mathrm{H}$ & -4.684593 & 1.221504 & -1.300323 \\
\hline $\mathrm{H}$ & -5.423676 & 0.974484 & 0.277047 \\
\hline $\mathrm{H}$ & 3.051639 & 1.583737 & 1.243298 \\
\hline $\mathrm{H}$ & 3.256502 & 2.724356 & -0.108985 \\
\hline $\mathrm{H}$ & 2.216592 & 3.134983 & 1.268102 \\
\hline $\mathrm{H}$ & 0.131254 & 3.513914 & 0.057760 \\
\hline $\mathrm{H}$ & 1.106216 & 3.424976 & -1.422954 \\
\hline $\mathrm{H}$ & -0.373658 & 2.455657 & -1.266947 \\
\hline $\mathrm{H}$ & 1.762147 & 1.008338 & -2.118182 \\
\hline $\mathrm{H}$ & 1.592473 & -3.423643 & -1.289346 \\
\hline $\mathrm{H}$ & 3.537292 & 0.137234 & -0.144500 \\
\hline $\mathrm{H}$ & -0.556472 & 0.288577 & -1.478254 \\
\hline $\mathrm{H}$ & -0.284911 & -2.027004 & -2.318672 \\
\hline $\mathrm{H}$ & 3.049875 & -2.241445 & 0.394721 \\
\hline
\end{tabular}

Sum of Energies $=-773.3595043$

\section{TS}




\begin{tabular}{|c|c|c|c|}
\hline $\mathrm{C}$ & 1.671168 & -2.220614 & 0.205154 \\
\hline C & 0.967303 & -2.633335 & -0.929333 \\
\hline $\mathrm{C}$ & 0.138009 & -1.723180 & -1.583282 \\
\hline C & 0.030674 & -0.429974 & -1.069203 \\
\hline C & 2.012935 & 0.458769 & -1.128955 \\
\hline $\mathrm{C}$ & 2.912719 & -0.527421 & -0.721648 \\
\hline C & 1.127857 & -1.162078 & 1.078882 \\
\hline C & 0.116112 & -0.184136 & 0.418963 \\
\hline $\mathrm{O}$ & 1.449567 & -1.034706 & 2.250981 \\
\hline C & -1.252328 & -0.377891 & 1.129341 \\
\hline C & -2.325944 & 0.556239 & 0.644645 \\
\hline C & -3.518322 & 0.245155 & 0.111332 \\
\hline C & -4.025537 & -1.156032 & -0.130173 \\
\hline C & -4.486599 & 1.333964 & -0.289312 \\
\hline 0 & 0.502902 & 1.163012 & 0.653036 \\
\hline C & 1.598569 & 1.607518 & -0.201024 \\
\hline C & 2.711893 & 2.072581 & 0.751259 \\
\hline C & 1.082120 & 2.796127 & -1.022054 \\
\hline $\mathrm{H}$ & 3.366811 & -1.176602 & -1.462954 \\
\hline $\mathrm{H}$ & -1.542210 & -1.428177 & 1.029572 \\
\hline $\mathrm{H}$ & -1.048869 & -0.200103 & 2.193774 \\
\hline $\mathrm{H}$ & -2.082450 & 1.610748 & 0.763039 \\
\hline $\mathrm{H}$ & -3.301102 & -1.932188 & 0.126429 \\
\hline $\mathrm{H}$ & -4.938951 & -1.343467 & 0.451726 \\
\hline $\mathrm{H}$ & -4.300010 & -1.290105 & -1.185872 \\
\hline $\mathrm{H}$ & -4.080864 & 2.332220 & -0.097638 \\
\hline $\mathrm{H}$ & -4.740138 & 1.267117 & -1.357046 \\
\hline $\mathrm{H}$ & -5.434796 & 1.241774 & 0.259286 \\
\hline $\mathrm{H}$ & 2.997429 & 1.275296 & 1.441693 \\
\hline $\mathrm{H}$ & 3.596461 & 2.405651 & 0.197283 \\
\hline $\mathrm{H}$ & 2.342828 & 2.908213 & 1.354964 \\
\hline $\mathrm{H}$ & 0.655873 & 3.550989 & -0.354156 \\
\hline $\mathrm{H}$ & 1.896274 & 3.258585 & -1.592507 \\
\hline $\mathrm{H}$ & 0.302093 & 2.486089 & -1.725276 \\
\hline $\mathrm{H}$ & 1.921672 & 0.646357 & -2.194955 \\
\hline $\mathrm{H}$ & 1.192721 & -3.595953 & -1.382588 \\
\hline $\mathrm{H}$ & 3.460150 & -0.438274 & 0.208884 \\
\hline $\mathrm{H}$ & 2.405369 & -2.871321 & 0.673040 \\
\hline $\mathrm{H}$ & -0.275911 & -1.962365 & -2.559407 \\
\hline $\mathrm{H}$ & -0.575918 & 0.294925 & -1.606989 \\
\hline
\end{tabular}

Sum of Energies $=-773.288133$

6
C
0.036240
0.025936
0.007353
$-0.018159$
C
0.025936
0.022065
1.435379 


$\begin{array}{lrrr}\mathrm{C} & 1.174992 & 0.026297 & 2.138939 \\ \mathrm{C} & 2.511197 & -0.040444 & 1.461083 \\ \mathrm{C} & 2.486667 & 0.237017 & -0.041967 \\ \mathrm{C} & 1.184414 & 0.086645 & -0.742748 \\ \mathrm{O} & 1.166087 & -0.037355 & -2.100814 \\ \mathrm{C} & 1.298537 & 1.096759 & -3.022010 \\ \mathrm{C} & 2.606499 & 0.886877 & -3.801546 \\ \mathrm{C} & 1.301226 & 2.448973 & -2.294668 \\ \mathrm{C} & 0.120258 & 1.037545 & -3.975584 \\ \mathrm{C} & -0.859583 & 0.131342 & -3.984763 \\ \mathrm{O} & 3.515139 & 0.545469 & -0.636121 \\ \mathrm{C} & 3.133809 & -1.472430 & 1.723746 \\ \mathrm{C} & 4.565834 & -1.611604 & 1.294486 \\ \mathrm{C} & 5.082717 & -2.451188 & 0.380784 \\ \mathrm{C} & 4.289341 & -3.434495 & -0.445115 \\ \mathrm{C} & 6.565892 & -2.450469 & 0.092561 \\ \mathrm{H} & 2.720713 & 1.656396 & -4.574823 \\ \mathrm{H} & 3.457196 & 0.932642 & -3.117016 \\ \mathrm{H} & 2.595131 & -0.093142 & -4.289431 \\ \mathrm{H} & 0.390913 & 2.587113 & -1.702480 \\ \mathrm{H} & 2.176491 & 2.544499 & -1.646020 \\ \mathrm{H} & 1.342421 & 3.253368 & -3.037398 \\ \mathrm{H} & 0.124275 & 1.829096 & -4.726741 \\ \mathrm{H} & -1.654435 & 0.174631 & -4.725052 \\ \mathrm{H} & -0.888740 & -0.681383 & -3.265462 \\ \mathrm{H} & 3.064491 & -1.634202 & 2.808751 \\ \mathrm{H} & 2.485549 & -2.220172 & 1.257070 \\ \mathrm{H} & 5.255727 & -0.933097 & 1.799125 \\ \mathrm{H} & 4.417266 & -3.220497 & -1.515308 \\ \mathrm{H} & 4.653880 & -4.459040 & -0.283657 \\ \mathrm{H} & 3.217720 & -3.423575 & -0.232145 \\ \mathrm{H} & 7.003654 & -3.445083 & 0.260102 \\ \mathrm{H} & 6.756761 & -2.195363 & -0.959583 \\ \mathrm{H} & -0.103750 & -1.730516 & 0.718074 \\ \mathrm{H} & -936628 & 0.023471 & 1.940819 \\ \mathrm{H} & 1.162404 & 0.012365 & 3.227287 \\ \mathrm{H} & 3.204341 & 0.687204 & 1.904134\end{array}$

Sum of Energies $=-773.349195$ 


\begin{tabular}{|c|c|c|c|}
\hline $\mathrm{C}$ & -0.753550 & 2.151386 & 0.573564 \\
\hline $\mathrm{C}$ & 0.143302 & 3.209026 & 0.271505 \\
\hline $\mathrm{C}$ & 1.281102 & 2.961568 & -0.462729 \\
\hline $\mathrm{C}$ & 1.592119 & 1.634990 & -0.862362 \\
\hline $\mathrm{C}$ & 0.660794 & 0.548172 & -0.646030 \\
\hline $\mathrm{C}$ & -0.515938 & 0.860909 & 0.151777 \\
\hline 0 & -1.374210 & -0.144383 & 0.520538 \\
\hline $\mathrm{C}$ & -2.381353 & -0.677082 & -0.392227 \\
\hline $\mathrm{C}$ & -2.021345 & -2.152517 & -0.633069 \\
\hline $\mathrm{C}$ & -2.444360 & 0.083893 & -1.725080 \\
\hline $\mathrm{C}$ & -3.723996 & -0.585316 & 0.307802 \\
\hline $\mathrm{C}$ & -3.950685 & -0.093301 & 1.524201 \\
\hline 0 & 0.916502 & -0.627680 & -1.059532 \\
\hline $\mathrm{C}$ & 2.434094 & -1.526939 & 0.390831 \\
\hline $\mathrm{C}$ & 2.714837 & -2.786959 & -0.375837 \\
\hline $\mathrm{C}$ & 1.460217 & -1.660343 & 1.527190 \\
\hline $\mathrm{C}$ & 3.283426 & -0.430442 & 0.246959 \\
\hline $\mathrm{C}$ & 3.026496 & 0.837875 & 0.751638 \\
\hline $\mathrm{H}$ & -2.782534 & -2.645692 & -1.249123 \\
\hline $\mathrm{H}$ & -1.052855 & -2.209227 & -1.135684 \\
\hline $\mathrm{H}$ & -1.960770 & -2.679525 & 0.324528 \\
\hline $\mathrm{H}$ & -2.650681 & 1.146726 & -1.569066 \\
\hline $\mathrm{H}$ & -1.508014 & -0.022437 & -2.279632 \\
\hline $\mathrm{H}$ & -3.252073 & -0.331971 & -2.337093 \\
\hline $\mathrm{H}$ & -4.554425 & -0.987206 & -0.274568 \\
\hline $\mathrm{H}$ & -4.951330 & -0.077946 & 1.947744 \\
\hline $\mathrm{H}$ & -3.143266 & 0.299201 & 2.133603 \\
\hline $\mathrm{H}$ & 3.074984 & -3.577305 & 0.298744 \\
\hline $\mathrm{H}$ & 1.792746 & -3.150752 & -0.840343 \\
\hline $\mathrm{H}$ & 3.460643 & -2.633810 & -1.160623 \\
\hline $\mathrm{H}$ & 1.042851 & -0.705208 & 1.850165 \\
\hline $\mathrm{H}$ & 0.627727 & -2.311454 & 1.253908 \\
\hline $\mathrm{H}$ & 1.975345 & -2.110077 & 2.389422 \\
\hline $\mathrm{H}$ & 4.068897 & -0.515822 & -0.502704 \\
\hline $\mathrm{H}$ & 3.743295 & 1.636171 & 0.591350 \\
\hline $\mathrm{H}$ & 2.329722 & 1.004550 & 1.564818 \\
\hline $\mathrm{H}$ & -0.092744 & 4.215889 & 0.604991 \\
\hline $\mathrm{H}$ & 2.364124 & 1.462697 & -1.604364 \\
\hline $\mathrm{H}$ & 1.958605 & 3.771256 & -0.722897 \\
\hline $\mathrm{H}$ & -1.659529 & 2.348937 & 1.139966 \\
\hline
\end{tabular}

Sum of Energies $=-773.289796$ 


\begin{tabular}{|c|c|c|c|}
\hline $\mathrm{C}$ & 1.010933 & -0.515758 & 1.585293 \\
\hline $\mathrm{C}$ & -0.061554 & -1.195130 & 1.162199 \\
\hline $\mathrm{C}$ & -0.426151 & -0.894739 & -0.274930 \\
\hline $\mathrm{C}$ & -0.684176 & 0.647901 & -0.378114 \\
\hline $\mathrm{C}$ & 0.496977 & 1.438636 & 0.273823 \\
\hline $\mathrm{C}$ & 1.596161 & 0.427748 & 0.561440 \\
\hline $\mathrm{C}$ & -3.218090 & 0.634604 & -0.575925 \\
\hline $\mathrm{C}$ & -4.248999 & -0.136921 & -0.194924 \\
\hline $\mathrm{C}$ & -4.428927 & -0.723245 & 1.184636 \\
\hline $\mathrm{C}$ & -5.360938 & -0.469694 & -1.162447 \\
\hline $\mathrm{C}$ & -2.033387 & 1.094201 & 0.229116 \\
\hline 0 & 0.504243 & 2.637097 & 0.442117 \\
\hline 0 & 2.984278 & 0.726133 & 0.748833 \\
\hline $\mathrm{C}$ & 3.353207 & -0.259034 & -0.301376 \\
\hline $\mathrm{C}$ & 0.833863 & -1.308440 & -1.136773 \\
\hline $\mathrm{C}$ & 1.877426 & -0.239866 & -0.813007 \\
\hline $\mathrm{C}$ & 3.842450 & -1.559974 & 0.324725 \\
\hline $\mathrm{C}$ & 4.384687 & 0.349902 & -1.238534 \\
\hline $\mathrm{H}$ & -0.681800 & 0.944747 & -1.438460 \\
\hline $\mathrm{H}$ & -3.221899 & 0.994074 & -1.607619 \\
\hline $\mathrm{H}$ & -3.600778 & -0.502762 & 1.861916 \\
\hline $\mathrm{H}$ & -5.350222 & -0.344041 & 1.648596 \\
\hline $\mathrm{H}$ & -4.538927 & -1.815261 & 1.129434 \\
\hline $\mathrm{H}$ & -5.192877 & -0.024427 & -2.148113 \\
\hline $\mathrm{H}$ & -5.462923 & -1.556560 & -1.291742 \\
\hline $\mathrm{H}$ & -6.330361 & -0.110690 & -0.788824 \\
\hline $\mathrm{H}$ & -2.014844 & 2.191879 & 0.263674 \\
\hline $\mathrm{H}$ & -2.088636 & 0.753343 & 1.268129 \\
\hline $\mathrm{H}$ & 1.829726 & 0.541540 & -1.579369 \\
\hline $\mathrm{H}$ & 4.088540 & -2.291494 & -0.454481 \\
\hline $\mathrm{H}$ & 4.746081 & -1.370666 & 0.914399 \\
\hline $\mathrm{H}$ & 3.089123 & -2.000777 & 0.983009 \\
\hline $\mathrm{H}$ & 4.034280 & 1.311431 & -1.625584 \\
\hline $\mathrm{H}$ & 5.328334 & 0.519667 & -0.707944 \\
\hline $\mathrm{H}$ & 4.582597 & -0.320892 & -2.083285 \\
\hline $\mathrm{H}$ & -0.602621 & -1.921569 & 1.762457 \\
\hline $\mathrm{H}$ & 1.138044 & -2.323445 & -0.863530 \\
\hline $\mathrm{H}$ & 1.465630 & -0.571807 & 2.569856 \\
\hline $\mathrm{H}$ & 0.589802 & -1.311483 & -2.204894 \\
\hline $\mathrm{H}$ & -1.315328 & -1.437785 & -0.606435 \\
\hline
\end{tabular}

Sum of Energies $=-773.318719$ 


\section{TS}

$\begin{array}{lrrr}\mathrm{C} & -0.926659 & -1.074674 & -1.346049 \\ \mathrm{C} & 0.136658 & -1.625995 & -0.634062 \\ \mathrm{C} & 0.608259 & -0.904070 & 0.471914 \\ \mathrm{C} & 0.699076 & 0.602348 & 0.313230 \\ \mathrm{C} & -0.515055 & 1.191645 & -0.433307 \\ \mathrm{C} & -1.455689 & 0.147134 & -0.900276 \\ \mathrm{C} & 3.247344 & 0.792098 & 0.390866 \\ \mathrm{C} & 4.297293 & 0.001494 & 0.116643 \\ \mathrm{C} & 4.438067 & -0.851418 & -1.120839 \\ \mathrm{C} & 5.469676 & -0.078926 & 1.066278 \\ \mathrm{C} & 2.006855 & 1.028872 & -0.425464 \\ \mathrm{O} & -0.669498 & 2.394212 & -0.581633 \\ \mathrm{O} & -2.806312 & 0.421514 & -1.092587 \\ \mathrm{C} & -3.312404 & -0.072924 & 0.199100 \\ \mathrm{C} & -2.043366 & -0.014125 & 1.091002 \\ \mathrm{C} & -3.887045 & -1.475857 & 0.021649 \\ \mathrm{C} & -4.372864 & 0.914992 & 0.678187 \\ \mathrm{H} & 3.279202 & 1.355873 & 1.326058 \\ \mathrm{H} & 3.560482 & -0.822975 & -1.770379 \\ \mathrm{H} & 5.306734 & -0.533082 & -1.714235 \\ \mathrm{H} & 4.619710 & -1.900139 & -0.847365 \\ \mathrm{H} & 5.327863 & 0.556034 & 1.946492 \\ \mathrm{H} & 5.631057 & -1.110166 & 1.411143 \\ \mathrm{H} & 6.401085 & 0.230561 & 0.571351 \\ \mathrm{H} & 1.898994 & 2.103216 & -0.626683 \\ \mathrm{H} & 2.050077 & 0.524913 & -1.395238 \\ \mathrm{H} & -1.898893 & 0.989723 & 1.487778 \\ \mathrm{H} & -4.292065 & -1.845385 & 0.970697 \\ \mathrm{H} & -4.693698 & -1.452925 & -0.718004 \\ \mathrm{H} & -3.127618 & -2.183651 & -0.322965 \\ \mathrm{H} & -3.951588 & 1.923026 & 0.734478 \\ \mathrm{H} & -5.216110 & 0.934480 & -0.020359 \\ \mathrm{H} & -4.746659 & 0.628053 & 1.667739 \\ \mathrm{H} & 0.417544 & -2.664501 & -0.791582 \\ \mathrm{C} & -1.246509 & -0.999292 & 1.677183 \\ \mathrm{H} & -0.736170 & -0.767624 & 2.608405 \\ \mathrm{H} & -1.461122 & -2.052890 & 1.530169 \\ \mathrm{H} & -1.502264 & -1.659032 & -2.058225 \\ \mathrm{H} & -362610 & -1.356963 & 1.111659 \\ \mathrm{H} & & 1.089839 & 1.296488\end{array}$

Sum of Energies $=-773.263268$ 
Gambogin System

8

\begin{tabular}{|c|c|c|c|}
\hline C & 2.670611 & -0.923634 & -0.101893 \\
\hline $\mathrm{C}$ & 2.130696 & 0.362278 & 0.119158 \\
\hline $\mathrm{O}$ & 0.786305 & 0.611336 & 0.087458 \\
\hline $\mathrm{C}$ & -0.101005 & -0.399754 & -0.145222 \\
\hline $\mathrm{C}$ & 0.323696 & -1.714057 & -0.356637 \\
\hline $\mathrm{C}$ & 1.767982 & -2.062324 & -0.365239 \\
\hline $\mathrm{O}$ & 2.132097 & -3.216468 & -0.574550 \\
\hline C & -0.645238 & -2.707332 & -0.561798 \\
\hline C & -1.991841 & -2.393819 & -0.520068 \\
\hline $\mathrm{C}$ & -2.415908 & -1.072506 & -0.278382 \\
\hline $\mathrm{C}$ & -1.467413 & -0.041792 & -0.147654 \\
\hline $\mathrm{O}$ & -1.827490 & 1.249164 & 0.123598 \\
\hline $\mathrm{C}$ & -2.007972 & 2.233591 & -0.943925 \\
\hline $\mathrm{C}$ & -3.421472 & 2.098470 & -1.532609 \\
\hline C & -0.956924 & 2.089559 & -2.056688 \\
\hline $\mathrm{C}$ & -1.856600 & 3.585370 & -0.278738 \\
\hline C & -1.474215 & 3.808698 & 0.976979 \\
\hline $\mathrm{O}$ & -3.755208 & -0.777318 & -0.265301 \\
\hline $\mathrm{C}$ & -4.526891 & -0.935060 & 0.964598 \\
\hline C & -3.943021 & -0.068029 & 2.091279 \\
\hline $\mathrm{C}$ & -4.578256 & -2.412719 & 1.394352 \\
\hline $\mathrm{C}$ & -5.929891 & -0.474798 & 0.631348 \\
\hline $\mathrm{C}$ & -6.397269 & -0.151527 & -0.572702 \\
\hline $\mathrm{H}$ & -0.298592 & -3.718658 & -0.745184 \\
\hline $\mathrm{H}$ & -2.747786 & -3.154661 & -0.679380 \\
\hline $\mathrm{H}$ & -3.601411 & 2.898326 & -2.260642 \\
\hline $\mathrm{H}$ & -3.538928 & 1.137559 & -2.040438 \\
\hline $\mathrm{H}$ & -4.177350 & 2.164531 & -0.746787 \\
\hline $\mathrm{H}$ & 0.053412 & 2.196131 & -1.658483 \\
\hline $\mathrm{H}$ & -1.045915 & 1.120345 & -2.559257 \\
\hline $\mathrm{H}$ & -1.117877 & 2.869498 & -2.808716 \\
\hline $\mathrm{H}$ & -2.090440 & 4.424986 & -0.933731 \\
\hline $\mathrm{H}$ & -1.391543 & 4.819965 & 1.366112 \\
\hline $\mathrm{H}$ & -1.234514 & 2.991429 & 1.648321 \\
\hline $\mathrm{H}$ & -4.580979 & -0.135083 & 2.979594 \\
\hline $\mathrm{H}$ & -2.942191 & -0.413643 & 2.370026 \\
\hline $\mathrm{H}$ & -3.872473 & 0.975472 & 1.779917 \\
\hline $\mathrm{H}$ & -4.967505 & -3.034252 & 0.582283 \\
\hline $\mathrm{H}$ & -3.587671 & -2.777753 & 1.682796 \\
\hline $\mathrm{H}$ & -5.242161 & -2.525050 & 2.258619 \\
\hline $\mathrm{H}$ & -6.593146 & -0.441675 & 1.495912 \\
\hline $\mathrm{H}$ & -7.431619 & 0.152804 & -0.707768 \\
\hline $\mathrm{H}$ & -5.766673 & -0.179884 & -1.454338 \\
\hline
\end{tabular}




$\begin{array}{lrrr}\mathrm{C} & 4.090673 & -1.032452 & -0.043449 \\ \mathrm{C} & 2.910460 & 1.482924 & 0.384383 \\ \mathrm{H} & 2.447145 & 2.447378 & 0.552113 \\ \mathrm{C} & 4.891099 & 0.082922 & 0.220746 \\ \mathrm{H} & 5.965031 & -0.021012 & 0.261184 \\ \mathrm{C} & 4.294536 & 1.336158 & 0.434439 \\ \mathrm{O} & 4.995992 & 2.469798 & 0.698709 \\ \mathrm{O} & 4.609452 & -2.260808 & -0.254329 \\ \mathrm{C} & 6.412980 & 2.401974 & 0.771389 \\ \mathrm{H} & 6.850479 & 2.078156 & -0.181627 \\ \mathrm{H} & 6.746348 & 3.416854 & 0.994203 \\ \mathrm{H} & 6.743535 & 1.728132 & 1.572090 \\ \mathrm{C} & 6.015651 & -2.440847 & -0.202175 \\ \mathrm{H} & 6.528466 & -1.844952 & -0.968575 \\ \mathrm{H} & 6.418711 & -2.187082 & 0.787004 \\ \mathrm{H} & 6.179657 & -3.501257 & -0.398870\end{array}$

Sum of Energies $=-1420.785125$

9

$\begin{array}{lrrr}\mathrm{C} & 2.766806 & 0.664613 & 0.294324 \\ \mathrm{C} & 2.483123 & -0.421361 & -0.564254 \\ \mathrm{O} & 1.202627 & -0.819565 & -0.828844 \\ \mathrm{C} & 0.164203 & -0.171519 & -0.249716 \\ \mathrm{C} & 0.310153 & 0.886063 & 0.592136 \\ \mathrm{C} & 1.661705 & 1.401371 & 0.933807 \\ \mathrm{O} & 1.785841 & 2.361589 & 1.690674 \\ \mathrm{C} & -0.863802 & 1.520504 & 1.175681 \\ \mathrm{C} & -2.105456 & 1.088736 & 0.918057 \\ \mathrm{C} & -2.427240 & -0.064244 & -0.005122 \\ \mathrm{C} & -1.161355 & -0.700400 & -0.656871 \\ \mathrm{O} & -1.244000 & -1.529835 & -1.548356 \\ \mathrm{O} & -3.203757 & -0.938343 & 0.845878 \\ \mathrm{C} & -3.268715 & -2.383833 & 0.786825 \\ \mathrm{C} & -3.812280 & -2.916532 & -0.549795 \\ \mathrm{C} & -4.284683 & -2.702027 & 1.903177 \\ \mathrm{C} & -1.936484 & -3.021138 & 1.154244 \\ \mathrm{C} & -1.039083 & -2.482645 & 1.980869 \\ \mathrm{C} & -3.300824 & 0.444495 & -1.201178 \\ \mathrm{C} & -4.619806 & 1.027039 & -0.778559 \\ \mathrm{C} & -5.125662 & 2.236345 & -1.070526 \\ \mathrm{C} & -4.434208 & 3.294801 & -1.894589 \\ \mathrm{C} & -6.497164 & 2.632419 & -0.575123 \\ \mathrm{H} & -0.669340 & 2.352890 & 1.843740 \\ \mathrm{H} & -2.969364 & 1.553247 & 1.382952 \\ \mathrm{H} & -3.985584 & -3.996376 & -0.469542\end{array}$




$\begin{array}{lrrr}\mathrm{H} & -4.771371 & -2.439342 & -0.776994 \\ \mathrm{H} & -3.117526 & -2.738477 & -1.370406 \\ \mathrm{H} & -3.947479 & -2.272671 & 2.850339 \\ \mathrm{H} & -5.265921 & -2.284398 & 1.652325 \\ \mathrm{H} & -4.387084 & -3.785083 & 2.028548 \\ \mathrm{H} & -1.753162 & -4.006397 & 0.726212 \\ \mathrm{H} & -0.119007 & -3.001784 & 2.234423 \\ \mathrm{H} & -1.192526 & -1.503115 & 2.426253 \\ \mathrm{H} & -2.703008 & 1.162803 & -1.772774 \\ \mathrm{H} & -3.457697 & -0.418350 & -1.860048 \\ \mathrm{H} & -5.226647 & 0.360902 & -0.167625 \\ \mathrm{H} & -5.023870 & 3.534265 & -2.790655 \\ \mathrm{H} & -4.345168 & 4.229712 & -1.324243 \\ \mathrm{H} & -3.432685 & 3.007560 & -2.222099 \\ \mathrm{H} & -7.168306 & 2.867237 & -1.413499 \\ \mathrm{H} & -6.962114 & 1.839991 & 0.019656 \\ \mathrm{H} & -6.447157 & 3.539176 & 0.044251 \\ \mathrm{C} & 4.145321 & 0.978515 & 0.482284 \\ \mathrm{C} & 3.464128 & -1.166685 & -1.208629 \\ \mathrm{H} & 3.191748 & -1.988947 & -1.858371 \\ \mathrm{C} & 4.798629 & -0.828599 & -1.000598 \\ \mathrm{C} & 5.145006 & 0.241411 & -0.156979 \\ \mathrm{H} & 6.183166 & 0.494683 & -0.002280 \\ \mathrm{O} & 4.418451 & 2.014588 & 1.302412 \\ \mathrm{O} & 5.707868 & -1.593017 & -1.658295 \\ \mathrm{C} & 5.769924 & 2.380837 & 1.534205 \\ \mathrm{H} & 6.269875 & 2.685681 & 0.605605 \\ \mathrm{H} & 5.727005 & 3.229443 & 2.218173 \\ \mathrm{H} & 6.334548 & 1.563735 & 2.001855 \\ \mathrm{C} & 7.091344 & -1.305257 & -1.507536 \\ \mathrm{H} & 7.613933 & -2.039835 & -2.122163 \\ \mathrm{H} & 7.333497 & -0.296130 & -1.864307 \\ \mathrm{H} & 7.413539 & -1.411205 & -0.463872\end{array}$

Sum of Energies $=-1420.763561$

\section{TS}

$\begin{array}{lrrr}C & -2.582725 & -0.764383 & -0.229826 \\ C & -2.095536 & 0.435109 & 0.335080 \\ O & -0.764921 & 0.733633 & 0.378618 \\ C & 0.153589 & -0.142543 & -0.115838 \\ C & -0.206071 & -1.352859 & -0.672331 \\ C & -1.633828 & -1.746441 & -0.791126 \\ O & -1.948731 & -2.811213 & -1.317811 \\ C & 0.809587 & -2.235030 & -1.143285 \\ C & 2.131932 & -1.904645 & -1.024471\end{array}$




\begin{tabular}{|c|c|c|c|}
\hline $\mathrm{C}$ & 2.533189 & -0.644236 & -0.478344 \\
\hline $\mathrm{C}$ & 1.540804 & 0.273275 & 0.074999 \\
\hline $\mathrm{O}$ & 1.840201 & 1.384395 & 0.582172 \\
\hline C & 2.271791 & 2.842064 & -1.285789 \\
\hline $\mathrm{C}$ & 2.920319 & 3.964105 & -0.528737 \\
\hline C & 0.810337 & 3.024728 & -1.578618 \\
\hline $\mathrm{C}$ & 3.053802 & 1.845876 & -1.850839 \\
\hline $\mathrm{C}$ & 2.572897 & 0.648741 & -2.381977 \\
\hline $\mathrm{O}$ & 3.865071 & -0.415900 & -0.210312 \\
\hline $\mathrm{C}$ & 4.475828 & -0.943641 & 1.022460 \\
\hline $\mathrm{C}$ & 5.772927 & -0.128096 & 1.119218 \\
\hline C & 4.790611 & -2.432202 & 0.845239 \\
\hline $\mathrm{C}$ & 3.601130 & -0.636056 & 2.218661 \\
\hline $\mathrm{C}$ & 3.214451 & -1.498385 & 3.158981 \\
\hline $\mathrm{H}$ & 0.486102 & -3.171950 & -1.583148 \\
\hline $\mathrm{H}$ & 2.909655 & -2.572226 & -1.382432 \\
\hline $\mathrm{H}$ & 2.740130 & 4.929319 & -1.023341 \\
\hline $\mathrm{H}$ & 2.482154 & 4.027012 & 0.473900 \\
\hline $\mathrm{H}$ & 3.999262 & 3.820376 & -0.424895 \\
\hline $\mathrm{H}$ & 0.330759 & 2.124613 & -1.966535 \\
\hline $\mathrm{H}$ & 0.274153 & 3.336721 & -0.678712 \\
\hline $\mathrm{H}$ & 0.689116 & 3.817618 & -2.332018 \\
\hline $\mathrm{H}$ & 4.123512 & 1.897755 & -1.665404 \\
\hline $\mathrm{H}$ & 3.274006 & -0.050278 & -2.822892 \\
\hline $\mathrm{H}$ & 1.540064 & 0.521686 & -2.686220 \\
\hline $\mathrm{H}$ & 6.364746 & -0.450326 & 1.981777 \\
\hline $\mathrm{H}$ & 6.368420 & -0.263399 & 0.209800 \\
\hline $\mathrm{H}$ & 5.542849 & 0.936055 & 1.227276 \\
\hline $\mathrm{H}$ & 3.879673 & -3.033464 & 0.781560 \\
\hline $\mathrm{H}$ & 5.370407 & -2.576803 & -0.071854 \\
\hline $\mathrm{H}$ & 5.385377 & -2.802701 & 1.686904 \\
\hline $\mathrm{H}$ & 3.294068 & 0.405472 & 2.277073 \\
\hline $\mathrm{H}$ & 2.607160 & -1.172128 & 3.999089 \\
\hline $\mathrm{H}$ & 3.478232 & -2.552264 & 3.139195 \\
\hline C & -2.921783 & 1.409993 & 0.887036 \\
\hline $\mathrm{H}$ & -2.498442 & 2.311205 & 1.312820 \\
\hline C & -3.996682 & -0.944712 & -0.211203 \\
\hline $\mathrm{C}$ & -4.841297 & 0.023393 & 0.338995 \\
\hline $\mathrm{H}$ & -5.909780 & -0.132119 & 0.343069 \\
\hline C & -4.297028 & 1.197311 & 0.886828 \\
\hline 0 & -4.464803 & -2.091791 & -0.748827 \\
\hline $\mathrm{O}$ & -5.046072 & 2.187276 & 1.441914 \\
\hline $\mathrm{C}$ & -5.861739 & -2.336527 & -0.756071 \\
\hline $\mathrm{H}$ & -5.983235 & -3.311047 & -1.231157 \\
\hline $\mathrm{H}$ & -6.270925 & -2.374239 & 0.262119 \\
\hline $\mathrm{H}$ & -6.402222 & -1.578227 & -1.337865 \\
\hline $\mathrm{C}$ & -6.457634 & 2.038387 & 1.489185 \\
\hline $\mathrm{H}$ & -6.751073 & 1.161607 & 2.080596 \\
\hline
\end{tabular}




$\begin{array}{llll}\mathrm{H} & -6.834168 & 2.941262 & 1.972849 \\ \mathrm{H} & -6.889174 & 1.959914 & 0.483054\end{array}$

Sum of Energies $=-1420.742467$

10

\begin{tabular}{|c|c|c|c|}
\hline $\mathrm{C}$ & -2.493148 & 0.462671 & -0.551557 \\
\hline $\mathrm{C}$ & -2.380350 & -0.844848 & -0.009131 \\
\hline 0 & -1.254099 & -1.605163 & -0.107494 \\
\hline C & -0.002161 & -1.019164 & -0.405776 \\
\hline C & -0.057195 & 0.345128 & -1.043156 \\
\hline $\mathrm{C}$ & -1.336285 & 1.092193 & -1.208799 \\
\hline $\mathrm{O}$ & -1.347272 & 2.151517 & -1.828514 \\
\hline $\mathrm{C}$ & 1.152672 & 0.829109 & -1.374185 \\
\hline $\mathrm{C}$ & 2.327091 & -0.019389 & -0.989736 \\
\hline $\mathrm{C}$ & 0.769396 & -1.963848 & -1.366381 \\
\hline $\mathrm{C}$ & 0.842407 & -0.788182 & 0.874287 \\
\hline $\mathrm{C}$ & 2.290754 & -0.330939 & 0.532755 \\
\hline $\mathrm{O}$ & 0.419322 & -0.933387 & 1.996923 \\
\hline $\mathrm{C}$ & 2.752554 & 0.784035 & 1.478707 \\
\hline $\mathrm{C}$ & 4.187917 & 1.184185 & 1.278297 \\
\hline $\mathrm{C}$ & 4.680759 & 2.404219 & 1.008724 \\
\hline $\mathrm{C}$ & 3.857996 & 3.659099 & 0.842611 \\
\hline $\mathrm{C}$ & 6.170502 & 2.615095 & 0.867769 \\
\hline $\mathrm{O}$ & 3.127679 & -1.485572 & 0.702018 \\
\hline $\mathrm{C}$ & 3.254045 & -2.206922 & -0.555278 \\
\hline $\mathrm{C}$ & 2.989743 & -3.687412 & -0.264378 \\
\hline $\mathrm{C}$ & 4.694873 & -2.040615 & -1.064422 \\
\hline $\mathrm{H}$ & 1.260832 & 1.803249 & -1.840492 \\
\hline $\mathrm{H}$ & 3.278673 & 0.445414 & -1.253040 \\
\hline $\mathrm{H}$ & 0.263157 & -1.919448 & -2.335517 \\
\hline $\mathrm{H}$ & 0.683303 & -2.989042 & -1.002215 \\
\hline $\mathrm{H}$ & 2.067849 & 1.630151 & 1.363072 \\
\hline $\mathrm{H}$ & 2.602720 & 0.402812 & 2.497701 \\
\hline $\mathrm{H}$ & 4.896356 & 0.363724 & 1.382820 \\
\hline $\mathrm{H}$ & 2.781515 & 3.489765 & 0.918261 \\
\hline $\mathrm{H}$ & 4.132761 & 4.403650 & 1.602895 \\
\hline $\mathrm{H}$ & 4.056848 & 4.126822 & -0.131687 \\
\hline $\mathrm{H}$ & 6.728805 & 1.681880 & 0.991324 \\
\hline $\mathrm{H}$ & 6.420604 & 3.035595 & -0.116673 \\
\hline $\mathrm{H}$ & 6.540229 & 3.333474 & 1.613137 \\
\hline $\mathrm{H}$ & 2.058228 & -3.831177 & 0.288217 \\
\hline $\mathrm{H}$ & 2.947077 & -4.271337 & -1.191167 \\
\hline $\mathrm{H}$ & 3.799849 & -4.089110 & 0.353090 \\
\hline $\mathrm{H}$ & 5.396730 & -2.359257 & -0.287134 \\
\hline $\mathrm{H}$ & 4.865845 & -2.657641 & -1.954467 \\
\hline
\end{tabular}




$\begin{array}{lrrr}\mathrm{H} & 4.923761 & -1.001154 & -1.316319 \\ \mathrm{C} & 2.239762 & -1.498303 & -1.503585 \\ \mathrm{H} & 2.566228 & -1.587531 & -2.543331 \\ \mathrm{C} & -3.447578 & -1.484960 & 0.609483 \\ \mathrm{H} & -3.322607 & -2.481661 & 1.013568 \\ \mathrm{C} & -3.758909 & 1.105320 & -0.421027 \\ \mathrm{C} & -4.836587 & 0.470959 & 0.202933 \\ \mathrm{H} & -5.785842 & 0.977183 & 0.293277 \\ \mathrm{C} & -4.671559 & -0.825931 & 0.712939 \\ \mathrm{O} & -3.851625 & 2.354859 & -0.925498 \\ \mathrm{O} & -5.659085 & -1.521640 & 1.333155 \\ \mathrm{C} & -6.931961 & -0.913062 & 1.498124 \\ \mathrm{H} & -7.545217 & -1.647696 & 2.022482 \\ \mathrm{H} & -6.865303 & 0.001046 & 2.101555 \\ \mathrm{H} & -7.394694 & -0.678796 & 0.530933 \\ \mathrm{C} & -5.076376 & 3.062141 & -0.812503 \\ \mathrm{H} & -5.889874 & 2.547644 & -1.340402 \\ \mathrm{H} & -5.360787 & 3.212664 & 0.237048 \\ \mathrm{H} & -4.896592 & 4.030386 & -1.281949\end{array}$

Sum of Energies $=-1420.803756$

\section{TS}

$\begin{array}{lrrr}\mathrm{C} & 2.565782 & 0.660028 & 0.229094 \\ \mathrm{C} & 2.420656 & -0.516416 & -0.541967 \\ \mathrm{O} & 1.197852 & -1.033313 & -0.870083 \\ \mathrm{C} & 0.060393 & -0.512969 & -0.325989 \\ \mathrm{C} & 0.082061 & 0.682612 & 0.417337 \\ \mathrm{C} & 1.369008 & 1.365660 & 0.724685 \\ \mathrm{O} & 1.375025 & 2.421028 & 1.352053 \\ \mathrm{C} & -1.101045 & 1.088135 & 1.032203 \\ \mathrm{C} & -2.250770 & 0.314593 & 0.874164 \\ \mathrm{C} & -0.553476 & -1.950151 & 1.298376 \\ \mathrm{C} & -1.176734 & -0.959413 & -1.022239 \\ \mathrm{C} & -2.504760 & -0.432218 & -0.416069 \\ \mathrm{O} & -1.158694 & -1.690277 & -1.995577 \\ \mathrm{C} & -3.187804 & 0.450111 & -1.496768 \\ \mathrm{C} & -4.536154 & 0.972486 & -1.085566 \\ \mathrm{C} & -4.939504 & 2.250262 & -0.998408 \\ \mathrm{C} & -4.080981 & 3.457172 & -1.289819 \\ \mathrm{C} & -6.358417 & 2.579165 & -0.596073 \\ \mathrm{O} & -3.384716 & -1.520703 & -0.164400 \\ \mathrm{C} & -3.090967 & -2.221422 & 1.079254 \\ \mathrm{C} & -2.916448 & -3.700209 & 0.699066 \\ \mathrm{C} & -4.306630 & -2.061771 & 2.001795 \\ \mathrm{H} & -1.049678 & 1.904870 & 1.745256\end{array}$




\begin{tabular}{|c|c|c|c|}
\hline $\mathrm{H}$ & -3.155876 & 0.630794 & 1.386759 \\
\hline $\mathrm{H}$ & 0.297381 & -1.700990 & 1.925078 \\
\hline $\mathrm{H}$ & -0.391715 & -2.777127 & 0.617149 \\
\hline $\mathrm{H}$ & -2.497923 & 1.255340 & -1.766719 \\
\hline $\mathrm{H}$ & -3.286400 & -0.197328 & -2.378293 \\
\hline $\mathrm{H}$ & -5.258809 & 0.194063 & -0.846590 \\
\hline $\mathrm{H}$ & -3.047407 & 3.208264 & -1.540014 \\
\hline $\mathrm{H}$ & -4.499122 & 4.036412 & -2.125042 \\
\hline $\mathrm{H}$ & -4.060940 & 4.133863 & -0.424400 \\
\hline $\mathrm{H}$ & -6.945692 & 1.677690 & -0.395030 \\
\hline $\mathrm{H}$ & -6.379603 & 3.209013 & 0.304671 \\
\hline $\mathrm{H}$ & -6.870568 & 3.149800 & -1.383809 \\
\hline $\mathrm{H}$ & -2.141822 & -3.822479 & -0.061649 \\
\hline $\mathrm{H}$ & -2.660536 & -4.308128 & 1.573672 \\
\hline $\mathrm{H}$ & -3.853410 & -4.075822 & 0.274894 \\
\hline $\mathrm{H}$ & -5.212023 & -2.385855 & 1.479458 \\
\hline $\mathrm{H}$ & -4.191464 & -2.672386 & 2.905082 \\
\hline $\mathrm{H}$ & -4.445167 & -1.019635 & 2.306563 \\
\hline $\mathrm{C}$ & -1.842889 & -1.593874 & 1.717305 \\
\hline $\mathrm{H}$ & -1.958567 & -1.233424 & 2.735691 \\
\hline C & 3.498715 & -1.230789 & -1.050747 \\
\hline $\mathrm{H}$ & 3.335423 & -2.122631 & -1.642810 \\
\hline C & 3.900883 & 1.096667 & 0.474755 \\
\hline C & 4.788158 & -0.771602 & -0.790369 \\
\hline $\mathrm{C}$ & 4.996659 & 0.388961 & -0.027066 \\
\hline $\mathrm{H}$ & 5.999895 & 0.735166 & 0.171380 \\
\hline $\mathrm{O}$ & 4.039996 & 2.216130 & 1.215556 \\
\hline $\mathrm{O}$ & 5.793213 & -1.517308 & -1.316988 \\
\hline $\mathrm{C}$ & 5.341151 & 2.709395 & 1.494661 \\
\hline $\mathrm{H}$ & 5.187891 & 3.610067 & 2.090673 \\
\hline $\mathrm{H}$ & 5.932031 & 1.986365 & 2.072041 \\
\hline $\mathrm{H}$ & 5.879907 & 2.968926 & 0.574151 \\
\hline C & 7.138595 & -1.114605 & -1.100249 \\
\hline $\mathrm{H}$ & 7.334603 & -0.121726 & -1.524393 \\
\hline $\mathrm{H}$ & 7.390867 & -1.112152 & -0.032211 \\
\hline $\mathrm{H}$ & 7.754431 & -1.854241 & -1.614248 \\
\hline
\end{tabular}

Sum of Energies $=-1420.740769$

11

$\begin{array}{lrrr}\text { C } & 2.476110 & -0.777002 & -0.161936 \\ \mathrm{C} & 1.832906 & 0.356234 & 0.375690 \\ \mathrm{O} & 0.468144 & 0.508887 & 0.314802 \\ \mathrm{C} & -0.298791 & -0.423327 & -0.288002 \\ \mathrm{C} & 0.215364 & -1.563093 & -0.822051 \\ \mathrm{C} & 1.671712 & -1.836182 & -0.805634\end{array}$




\begin{tabular}{|c|c|c|c|}
\hline $\mathrm{O}$ & 2.123160 & -2.867383 & -1.298092 \\
\hline C & -0.680371 & -2.535997 & -1.419935 \\
\hline C & -2.021301 & -2.370356 & -1.465745 \\
\hline C & -2.664702 & -1.158843 & -0.953087 \\
\hline C & -1.783616 & -0.122578 & -0.188822 \\
\hline $\mathrm{O}$ & -2.106985 & 1.229747 & -0.466318 \\
\hline $\mathrm{C}$ & -1.963903 & 1.879684 & -1.754377 \\
\hline $\mathrm{C}$ & -2.752981 & 1.186760 & -2.879684 \\
\hline C & -0.486552 & 2.031250 & -2.169513 \\
\hline $\mathrm{C}$ & -2.537822 & 3.271017 & -1.545925 \\
\hline C & -2.994212 & 3.786806 & -0.406876 \\
\hline 0 & -3.866749 & -0.961541 & -1.055645 \\
\hline $\mathrm{C}$ & -2.139518 & -0.292702 & 1.341755 \\
\hline C & -3.581073 & -0.046769 & 1.674023 \\
\hline C & -4.397724 & -0.830048 & 2.396672 \\
\hline C & -4.027598 & -2.165196 & 2.994416 \\
\hline $\mathrm{C}$ & -5.818853 & -0.400162 & 2.671992 \\
\hline $\mathrm{H}$ & -0.200256 & -3.417537 & -1.834550 \\
\hline $\mathrm{H}$ & -2.675858 & -3.098867 & -1.934594 \\
\hline $\mathrm{H}$ & -2.727988 & 1.817404 & -3.775614 \\
\hline $\mathrm{H}$ & -2.310460 & 0.221890 & -3.148578 \\
\hline $\mathrm{H}$ & -3.790865 & 1.025013 & -2.585210 \\
\hline $\mathrm{H}$ & 0.093050 & 2.500384 & -1.371339 \\
\hline $\mathrm{H}$ & -0.037280 & 1.065041 & -2.422454 \\
\hline $\mathrm{H}$ & -0.420565 & 2.663518 & -3.062024 \\
\hline $\mathrm{H}$ & -2.545077 & 3.875966 & -2.452924 \\
\hline $\mathrm{H}$ & -3.379332 & 4.802315 & -0.369488 \\
\hline $\mathrm{H}$ & -2.998389 & 3.217189 & 0.515516 \\
\hline $\mathrm{H}$ & -1.806031 & -1.285034 & 1.661220 \\
\hline $\mathrm{H}$ & -1.505628 & 0.443259 & 1.852889 \\
\hline $\mathrm{H}$ & -3.978796 & 0.887992 & 1.287600 \\
\hline $\mathrm{H}$ & -4.133027 & -2.143921 & 4.088351 \\
\hline $\mathrm{H}$ & -4.709055 & -2.947549 & 2.632837 \\
\hline $\mathrm{H}$ & -3.007315 & -2.482818 & 2.766255 \\
\hline $\mathrm{H}$ & -6.012819 & -0.333277 & 3.752202 \\
\hline $\mathrm{H}$ & -6.045054 & 0.571710 & 2.223288 \\
\hline $\mathrm{H}$ & -6.533358 & -1.133024 & 2.271579 \\
\hline $\mathrm{C}$ & 2.500631 & 1.401250 & 1.000692 \\
\hline $\mathrm{H}$ & 1.955805 & 2.249169 & 1.396821 \\
\hline $\mathrm{C}$ & 3.894868 & -0.814681 & -0.034989 \\
\hline $\mathrm{C}$ & 4.588707 & 0.225183 & 0.592037 \\
\hline $\mathrm{H}$ & 5.663927 & 0.178252 & 0.679726 \\
\hline $\mathrm{C}$ & 3.888603 & 1.328449 & 1.107115 \\
\hline $\mathrm{O}$ & 4.516244 & -1.895568 & -0.549189 \\
\hline $\mathrm{O}$ & 4.484843 & 2.377738 & 1.728418 \\
\hline $\mathrm{C}$ & 5.929737 & -1.994774 & -0.460961 \\
\hline $\mathrm{H}$ & 6.267940 & -2.017472 & 0.583100 \\
\hline $\mathrm{H}$ & 6.184367 & -2.938666 & -0.944602 \\
\hline
\end{tabular}




$\begin{array}{rrrr}\mathrm{H} & 6.425363 & -1.169374 & -0.988294 \\ \mathrm{C} & 5.898913 & 2.381950 & 1.873351 \\ \mathrm{H} & 6.139164 & 3.310468 & 2.393399 \\ \mathrm{H} & 6.244193 & 1.530440 & 2.473196 \\ \mathrm{H} & 6.402882 & 2.371244 & 0.898646\end{array}$

Sum of Energies $=-1420.771685$

\section{$11 \mathrm{TS}$}

$\begin{array}{lrrr}\mathrm{C} & 2.630374 & -0.764873 & -0.191431 \\ \mathrm{C} & 2.028109 & 0.457291 & 0.175422 \\ \mathrm{O} & 0.671504 & 0.646092 & 0.138543 \\ \mathrm{C} & -0.151843 & -0.365772 & -0.258720 \\ \mathrm{C} & 0.336457 & -1.603215 & -0.648340 \\ \mathrm{C} & 1.785939 & -1.893258 & -0.643716 \\ \mathrm{O} & 2.212832 & -2.991959 & -0.993910 \\ \mathrm{C} & -0.591021 & -2.592774 & -1.080727 \\ \mathrm{C} & -1.937390 & -2.354185 & -1.097097 \\ \mathrm{C} & -2.492156 & -1.073802 & -0.711045 \\ \mathrm{C} & -1.550925 & -0.077129 & -0.208489 \\ \mathrm{O} & -1.937934 & 1.200016 & 0.081709 \\ \mathrm{C} & -2.306882 & 2.179180 & -0.967088 \\ \mathrm{C} & -1.941605 & 1.694502 & -2.376000 \\ \mathrm{C} & -1.506131 & 3.430240 & -0.601898 \\ \mathrm{C} & -3.808612 & 2.353446 & -0.858425 \\ \mathrm{C} & -4.465010 & 3.500378 & -0.681295 \\ \mathrm{O} & -3.738353 & -0.852875 & -0.709594 \\ \mathrm{C} & -4.420188 & -1.207456 & 1.539128 \\ \mathrm{C} & -4.381003 & -2.698776 & 1.372400 \\ \mathrm{C} & -5.779689 & -0.580971 & 1.439063 \\ \mathrm{C} & -3.348958 & -0.459472 & 2.020463 \\ \mathrm{C} & -2.019664 & -0.857440 & 1.972580 \\ \mathrm{H} & -0.174414 & -3.544019 & -1.396951 \\ \mathrm{H} & -2.642106 & -3.099963 & -1.453276 \\ \mathrm{H} & -2.168823 & 2.491686 & -3.091227 \\ \mathrm{H} & -0.873624 & 1.462339 & -2.450492 \\ \mathrm{H} & -2.518076 & 0.811420 & -2.662804 \\ \mathrm{H} & -1.741804 & 3.749942 & 0.417305 \\ \mathrm{H} & -0.436663 & 3.209444 & -0.655660 \\ \mathrm{H} & -1.727895 & 4.253804 & -1.288579 \\ \mathrm{H} & -4.349222 & 1.414066 & -0.946166 \\ \mathrm{H} & -5.550974 & 3.520749 & -0.634048 \\ \mathrm{H} & -3.964961 & 4.460014 & -0.580575 \\ \mathrm{H} & -4.725265 & -3.176398 & 2.301974 \\ \mathrm{H} & -5.054312 & -3.012921 & 0.570157 \\ \mathrm{H} & -3.382631 & -3.081959 & 1.151839\end{array}$




$\begin{array}{rrrr}\mathrm{H} & -5.748182 & 0.498648 & 1.606457 \\ \mathrm{H} & -6.197536 & -0.768683 & 0.444045 \\ \mathrm{H} & -6.467708 & -1.031189 & 2.169614 \\ \mathrm{H} & -3.539051 & 0.588468 & 2.236114 \\ \mathrm{H} & -1.251223 & -0.196263 & 2.358271 \\ \mathrm{H} & -1.724822 & -1.889891 & 1.823413 \\ \mathrm{C} & 2.747772 & 1.568716 & 0.601224 \\ \mathrm{H} & 2.237788 & 2.484311 & 0.874110 \\ \mathrm{C} & 4.051382 & -0.820043 & -0.102581 \\ \mathrm{C} & 4.793015 & 0.286588 & 0.323198 \\ \mathrm{H} & 5.869276 & 0.224066 & 0.382754 \\ \mathrm{C} & 4.135474 & 1.476867 & 0.672616 \\ \mathrm{O} & 4.632422 & -1.988731 & -0.449011 \\ \mathrm{O} & 4.777989 & 2.598179 & 1.095555 \\ \mathrm{C} & 6.044268 & -2.109093 & -0.386732 \\ \mathrm{H} & 6.418156 & -1.962134 & 0.635157 \\ \mathrm{H} & 6.262867 & -3.128179 & -0.708898 \\ \mathrm{H} & 6.540783 & -1.399641 & -1.061798 \\ \mathrm{C} & 6.194902 & 2.583058 & 1.193314 \\ \mathrm{H} & 6.477920 & 3.577437 & 1.542488 \\ \mathrm{H} & 6.541032 & 1.833185 & 1.916156 \\ \mathrm{H} & 6.664091 & 2.393836 & 0.219352\end{array}$

Sum of Energies $=-1420.744703$

12

$\begin{array}{lrrr}\text { C } & 2.118073 & -0.055644 & 0.767088 \\ C & 1.638178 & -0.473924 & -0.498836 \\ \text { O } & 0.364051 & -0.892406 & -0.731584 \\ C & -0.640465 & -0.954904 & 0.282207 \\ C & -0.169975 & -0.534104 & 1.645573 \\ C & 1.215475 & -0.057049 & 1.926495 \\ \text { O } & 1.517030 & 0.291145 & 3.064477 \\ C & -1.111351 & -0.578211 & 2.603510 \\ C & -2.435839 & -1.125784 & 2.160819 \\ C & -2.136622 & -2.534029 & 1.546773 \\ C & -1.338575 & -2.366203 & 0.232095 \\ C & -1.882980 & -0.113630 & -0.158868 \\ C & -2.906978 & -0.201896 & 1.020940 \\ C & -2.748931 & 2.079040 & -1.141468 \\ C & -3.104245 & 3.344516 & -0.876455 \\ C & -2.389800 & 4.269432 & 0.078444 \\ C & -4.309360 & 3.957187 & -1.550303 \\ C & -2.213202 & -2.266283 & -1.070663 \\ C & -1.465396 & -2.796919 & -2.300968 \\ C & -3.596575 & -2.926032 & -0.998979\end{array}$




$\begin{array}{rrrr}\mathrm{C} & -1.566475 & 1.320215 & -0.598812 \\ \mathrm{O} & -3.983053 & 0.351371 & 1.009635 \\ \mathrm{O} & -2.422233 & -0.837510 & -1.262467 \\ \mathrm{H} & -0.904058 & -0.261553 & 3.620520 \\ \mathrm{H} & -3.192152 & -1.160911 & 2.946248 \\ \mathrm{H} & -1.543005 & -3.097035 & 2.273377 \\ \mathrm{H} & -0.591555 & -3.157220 & 0.130828 \\ \mathrm{H} & -3.351603 & 1.516688 & -1.851774 \\ \mathrm{H} & -1.540311 & 3.805382 & 0.585525 \\ \mathrm{H} & -2.016471 & 5.159279 & -0.448297 \\ \mathrm{H} & -3.081033 & 4.634499 & 0.850836 \\ \mathrm{H} & -4.794084 & 3.254768 & -2.235365 \\ \mathrm{H} & -5.054988 & 4.275880 & -0.808061 \\ \mathrm{H} & -4.034092 & 4.856032 & -2.120898 \\ \mathrm{H} & -0.468466 & -2.361786 & -2.376450 \\ \mathrm{H} & -1.372501 & -3.888540 & -2.251652 \\ \mathrm{H} & -2.027205 & -2.543499 & -3.206030 \\ \mathrm{H} & -4.088709 & -2.827468 & -1.971882 \\ \mathrm{H} & -3.520259 & -3.994348 & -0.764417 \\ \mathrm{H} & -4.241577 & -2.444780 & -0.259301 \\ \mathrm{H} & -3.063557 & -3.087323 & 1.388783 \\ \mathrm{H} & -1.099816 & 1.844756 & 0.241248 \\ \mathrm{H} & -0.803750 & 1.223058 & -1.383861 \\ \mathrm{C} & 3.483096 & 0.352632 & 0.837514 \\ \mathrm{C} & 2.449604 & -0.489518 & -1.629633 \\ \mathrm{H} & 2.051122 & -0.808446 & -2.584851 \\ \mathrm{C} & 4.305588 & 0.339325 & -0.292194 \\ \mathrm{H} & 5.335723 & 0.653504 & -0.218611 \\ \mathrm{C} & 3.778523 & -0.083000 & -1.522039 \\ \mathrm{O} & 3.929023 & 0.747695 & 2.048371 \\ \mathrm{O} & 4.496949 & -0.125758 & -2.673426 \\ \mathrm{C} & 5.276927 & 1.167758 & 2.192514 \\ \mathrm{H} & 5.493764 & 2.047748 & 1.573214 \\ \mathrm{H} & 5.387417 & 1.429997 & 3.245624 \\ \mathrm{H} & 5.979766 & 0.362672 & 1.941696 \\ \mathrm{C} & 5.863588 & 0.263046 & -2.648371 \\ \mathrm{H} & 5.225865 & 0.134817 & -3.669600 \\ \mathrm{H} & \mathrm{H} & 1.313604 & -2.352430 \\ \mathrm{H} & -0.372206 & -1.972743\end{array}$

Sum of Energies $=-1420.797936$ 


\section{$12 \mathrm{TS}$}

\begin{tabular}{|c|c|c|c|}
\hline C & 2.289001 & -0.697686 & -0.347168 \\
\hline C & 1.750582 & 0.343420 & 0.439739 \\
\hline $\mathrm{O}$ & 0.414332 & 0.643796 & 0.445055 \\
\hline C & -0.448155 & 0.069747 & -0.457640 \\
\hline C & -0.017025 & -1.037961 & -1.218670 \\
\hline C & 1.394982 & -1.499317 & -1.204804 \\
\hline 0 & 1.740501 & -2.471524 & -1.871151 \\
\hline C & -0.914232 & -1.568878 & -2.139277 \\
\hline C & -2.178306 & -0.988292 & -2.279050 \\
\hline $\mathrm{C}$ & -1.582190 & 1.008121 & -2.936192 \\
\hline $\mathrm{C}$ & -0.957689 & 1.633088 & -1.844688 \\
\hline C & -1.895639 & 0.190853 & 0.002724 \\
\hline C & -2.835764 & -0.353195 & -1.113316 \\
\hline C & -3.493212 & -0.467724 & 1.898150 \\
\hline C & -4.287917 & -1.451504 & 2.345508 \\
\hline $\mathrm{C}$ & -3.950361 & -2.922392 & 2.332175 \\
\hline C & -5.647534 & -1.131720 & 2.920420 \\
\hline C & -1.761247 & 2.443959 & -0.817071 \\
\hline C & -0.886440 & 3.533138 & -0.185845 \\
\hline $\mathrm{C}$ & -3.050211 & 3.070345 & -1.373523 \\
\hline $\mathrm{C}$ & -2.107772 & -0.592501 & 1.327949 \\
\hline $\mathrm{O}$ & -4.043102 & -0.198610 & -1.043611 \\
\hline 0 & -2.173873 & 1.551416 & 0.270663 \\
\hline $\mathrm{H}$ & -0.548544 & -2.318643 & -2.834564 \\
\hline $\mathrm{H}$ & -2.861629 & -1.334880 & -3.050048 \\
\hline $\mathrm{H}$ & -0.972447 & 0.673461 & -3.769466 \\
\hline $\mathrm{H}$ & 0.089904 & 1.900814 & -1.954504 \\
\hline $\mathrm{H}$ & -3.859702 & 0.554461 & 1.956805 \\
\hline $\mathrm{H}$ & -2.974258 & -3.145255 & 1.894348 \\
\hline $\mathrm{H}$ & -3.957715 & -3.332245 & 3.352185 \\
\hline $\mathrm{H}$ & -4.706512 & -3.485048 & 1.767177 \\
\hline $\mathrm{H}$ & -5.849360 & -0.056297 & 2.914237 \\
\hline $\mathrm{H}$ & -6.442703 & -1.629063 & 2.347106 \\
\hline $\mathrm{H}$ & -5.738037 & -1.491489 & 3.955618 \\
\hline $\mathrm{H}$ & 0.038298 & 3.111259 & 0.214142 \\
\hline $\mathrm{H}$ & -0.633507 & 4.299749 & -0.927605 \\
\hline $\mathrm{H}$ & -1.429336 & 4.013749 & 0.634215 \\
\hline $\mathrm{H}$ & -3.534601 & 3.648705 & -0.580164 \\
\hline $\mathrm{H}$ & -2.833229 & 3.743231 & -2.210687 \\
\hline $\mathrm{H}$ & -3.761561 & 2.307858 & -1.697402 \\
\hline $\mathrm{H}$ & -2.609983 & 1.238516 & -3.193314 \\
\hline $\mathrm{H}$ & -1.825681 & -1.636241 & 1.157277 \\
\hline $\mathrm{H}$ & -1.377368 & -0.170664 & 2.031476 \\
\hline $\mathrm{C}$ & 2.518238 & 1.130943 & 1.290069 \\
\hline $\mathrm{H}$ & 2.059140 & 1.911017 & 1.884687 \\
\hline C & 3.693711 & -0.913814 & -0.239809 \\
\hline
\end{tabular}




$\begin{array}{lrrr}\mathrm{C} & 4.482262 & -0.130622 & 0.608775 \\ \mathrm{H} & 5.544908 & -0.309618 & 0.676128 \\ \mathrm{C} & 3.888102 & 0.886791 & 1.372392 \\ \mathrm{O} & 4.209851 & -1.900994 & -1.000927 \\ \mathrm{O} & 4.576235 & 1.691297 & 2.222724 \\ \mathrm{C} & 5.977862 & 1.504990 & 2.366192 \\ \mathrm{H} & 6.503895 & 1.664272 & 1.416376 \\ \mathrm{H} & 6.302923 & 2.255077 & 3.088807 \\ \mathrm{H} & 6.213078 & 0.504614 & 2.750942 \\ \mathrm{C} & 5.602095 & -2.170708 & -0.942695 \\ \mathrm{H} & 6.195170 & -1.303766 & -1.262003 \\ \mathrm{H} & 5.912201 & -2.474047 & 0.065650 \\ \mathrm{H} & 5.766251 & -2.997077 & -1.635527\end{array}$

Sum of Energies $=-1420.741342$

\section{Pink Pathway (Claisen Intermediate)}

$\begin{array}{lrrr}\mathrm{C} & 2.161293 & 0.060420 & -0.613003 \\ \mathrm{C} & 1.653399 & -1.051015 & 0.107848 \\ \mathrm{O} & 0.331107 & -1.256838 & 0.349885 \\ \mathrm{C} & -0.631184 & -0.241831 & 0.051661 \\ \mathrm{C} & -0.209498 & 0.663848 & -1.061823 \\ \mathrm{C} & 1.227913 & 1.032540 & -1.207576 \\ \mathrm{O} & 1.548819 & 2.036423 & -1.840945 \\ \mathrm{C} & -1.141436 & 1.172293 & -1.898990 \\ \mathrm{C} & -2.518750 & 0.745669 & -1.864367 \\ \mathrm{C} & -2.960849 & -0.253441 & -1.048094 \\ \mathrm{C} & -1.980657 & -0.964488 & -0.180935 \\ \mathrm{O} & -2.230191 & -2.011430 & 0.392007 \\ \mathrm{O} & -4.199260 & -0.762089 & -1.248047 \\ \mathrm{C} & -5.219407 & -0.927233 & -0.179683 \\ \mathrm{C} & -5.450490 & -2.435708 & -0.035752 \\ \mathrm{C} & -6.454385 & -0.237646 & -0.764509 \\ \mathrm{C} & -4.765460 & -0.341282 & 1.141810 \\ \mathrm{C} & -5.218596 & 0.779212 & 1.706436 \\ \mathrm{C} & -0.896110 & 0.598320 & 1.372356 \\ \mathrm{C} & 0.305621 & 1.324488 & 1.907158 \\ \mathrm{C} & 0.596010 & 2.630508 & 1.782342 \\ \mathrm{C} & -0.240939 & 3.634753 & 1.029861 \\ \mathrm{C} & 1.866367 & 3.190513 & 2.374796 \\ \mathrm{H} & -0.826900 & 1.902525 & -2.638628 \\ \mathrm{H} & -3.227222 & 1.182033 & -2.563123 \\ \mathrm{H} & -6.264585 & -2.629874 & 0.671782 \\ \mathrm{H} & -5.723003 & -2.864091 & -1.005905 \\ \mathrm{H} & -4.540460 & -2.923871 & 0.318621 \\ \mathrm{H} & -6.276920 & 0.830642 & -0.921637\end{array}$




$\begin{array}{lrrr}\mathrm{H} & -6.693041 & -0.685297 & -1.733580 \\ \mathrm{H} & -7.317751 & -0.358480 & -0.102175 \\ \mathrm{H} & -4.023085 & -0.935789 & 1.668370 \\ \mathrm{H} & -4.849391 & 1.102715 & 2.676222 \\ \mathrm{H} & -5.969973 & 1.413232 & 1.244831 \\ \mathrm{H} & -1.729480 & 1.277202 & 1.167374 \\ \mathrm{H} & -1.242085 & -0.137882 & 2.108398 \\ \mathrm{H} & 1.012544 & 0.699199 & 2.450496 \\ \mathrm{H} & 0.265463 & 3.916360 & 0.096762 \\ \mathrm{H} & -1.232006 & 3.259945 & 0.763098 \\ \mathrm{H} & -0.368803 & 4.552986 & 1.618053 \\ \mathrm{H} & 2.499823 & 3.623430 & 1.588267 \\ \mathrm{H} & 1.648751 & 4.000704 & 3.084612 \\ \mathrm{H} & 2.447842 & 2.424285 & 2.896663 \\ \mathrm{C} & 3.575647 & 0.135219 & -0.768287 \\ \mathrm{C} & 2.483864 & -2.038909 & 0.628448 \\ \mathrm{H} & 2.059901 & -2.873976 & 1.172257 \\ \mathrm{C} & 4.418387 & -0.852421 & -0.249596 \\ \mathrm{H} & 5.487292 & -0.778215 & -0.380830 \\ \mathrm{C} & 3.861540 & -1.938644 & 0.442817 \\ \mathrm{O} & 4.050612 & 1.204695 & -1.444367 \\ \mathrm{O} & 4.598504 & -2.945430 & 0.980960 \\ \mathrm{C} & 5.446630 & 1.323586 & -1.665642 \\ \mathrm{H} & 5.573011 & 2.246462 & -2.233730 \\ \mathrm{H} & 6.001253 & 1.399120 & -0.721049 \\ \mathrm{H} & 5.836680 & 0.480052 & -2.249990 \\ \mathrm{C} & 6.011255 & -2.917705 & 0.837837 \\ \mathrm{H} & 6.447732 & -2.032764 & 1.318450 \\ \mathrm{H} & 6.376875 & -3.816231 & 1.337662 \\ \mathrm{H} & 6.309815 & -2.943346 & -0.217904\end{array}$

Sum of Energies $=-1420.762138$

\section{Pink Pathway (Claisen TS)}

$\begin{array}{lrrr}\text { C } & 2.583117 & -0.845560 & -0.091405 \\ \mathrm{C} & 2.076813 & 0.355741 & 0.458938 \\ \mathrm{O} & 0.742197 & 0.616442 & 0.561845 \\ \mathrm{C} & -0.172374 & -0.216533 & -0.037822 \\ \mathrm{C} & 0.211102 & -1.540113 & -0.411620 \\ \mathrm{C} & 1.648445 & -1.905876 & -0.521865 \\ \mathrm{O} & 1.983393 & -3.016258 & -0.926944 \\ \mathrm{C} & -0.773587 & -2.450800 & -0.736012 \\ \mathrm{C} & -2.139669 & -2.121443 & -0.594730 \\ \mathrm{C} & -2.543780 & -0.884125 & -0.132013 \\ \mathrm{C} & -1.568341 & 0.143246 & 0.233199 \\ \mathrm{O} & -1.879218 & 1.284393 & 0.643593\end{array}$




\begin{tabular}{|c|c|c|c|}
\hline $\mathrm{C}$ & -1.747430 & 2.698162 & -1.393308 \\
\hline C & -1.819428 & 4.054710 & -0.755321 \\
\hline $\mathrm{C}$ & -3.052668 & 2.151623 & -1.894391 \\
\hline C & -0.517702 & 2.122313 & -1.654513 \\
\hline C & -0.304831 & 0.791401 & -2.041070 \\
\hline 0 & -3.871352 & -0.565947 & -0.047480 \\
\hline C & -4.648595 & -0.857064 & 1.150701 \\
\hline C & -4.014754 & -0.208379 & 2.390894 \\
\hline $\mathrm{C}$ & -4.786909 & -2.378191 & 1.341658 \\
\hline C & -6.017813 & -0.261608 & 0.902653 \\
\hline C & -6.438944 & 0.320847 & -0.218063 \\
\hline $\mathrm{H}$ & -0.467896 & -3.430869 & -1.086735 \\
\hline $\mathrm{H}$ & -2.900522 & -2.849607 & -0.858464 \\
\hline $\mathrm{H}$ & -2.326770 & 4.774721 & -1.412984 \\
\hline $\mathrm{H}$ & -2.408373 & 3.990594 & 0.167223 \\
\hline $\mathrm{H}$ & -0.829656 & 4.445656 & -0.504013 \\
\hline $\mathrm{H}$ & -3.001008 & 1.100583 & -2.182118 \\
\hline $\mathrm{H}$ & -3.830947 & 2.253277 & -1.133804 \\
\hline $\mathrm{H}$ & -3.368475 & 2.727706 & -2.777416 \\
\hline $\mathrm{H}$ & 0.361156 & 2.684015 & -1.344710 \\
\hline $\mathrm{H}$ & 0.702911 & 0.477849 & -2.292819 \\
\hline $\mathrm{H}$ & -1.091685 & 0.185479 & -2.475951 \\
\hline $\mathrm{H}$ & -4.676312 & -0.333699 & 3.255565 \\
\hline $\mathrm{H}$ & -3.055641 & -0.679442 & 2.630711 \\
\hline $\mathrm{H}$ & -3.840010 & 0.854212 & 2.215558 \\
\hline $\mathrm{H}$ & -5.218782 & -2.838407 & 0.447541 \\
\hline $\mathrm{H}$ & -3.815237 & -2.838381 & 1.547605 \\
\hline $\mathrm{H}$ & -5.446145 & -2.592405 & 2.190174 \\
\hline $\mathrm{H}$ & -6.695104 & -0.355941 & 1.751753 \\
\hline $\mathrm{H}$ & -7.449879 & 0.710714 & -0.301058 \\
\hline $\mathrm{H}$ & -5.791554 & 0.423152 & -1.082197 \\
\hline $\mathrm{C}$ & 2.896287 & 1.366618 & 0.951545 \\
\hline $\mathrm{H}$ & 2.462655 & 2.266166 & 1.370569 \\
\hline C & 4.277976 & 1.197926 & 0.898442 \\
\hline $\mathrm{C}$ & 4.002141 & -0.975229 & -0.138242 \\
\hline C & 4.838539 & 0.033608 & 0.349130 \\
\hline $\mathrm{H}$ & 5.910792 & -0.084045 & 0.303685 \\
\hline $\mathrm{O}$ & 4.485163 & -2.114252 & -0.678849 \\
\hline 0 & 5.014254 & 2.225470 & 1.397304 \\
\hline $\mathrm{C}$ & 6.431406 & 2.128551 & 1. 381861 \\
\hline $\mathrm{H}$ & 6.795947 & 3.054723 & 1.829155 \\
\hline $\mathrm{H}$ & 6.783197 & 1.275861 & 1.976442 \\
\hline $\mathrm{H}$ & 6.818405 & 2.043499 & 0.358365 \\
\hline C & 5.888222 & -2.311638 & -0.748334 \\
\hline $\mathrm{H}$ & 6.373777 & -1.547795 & -1.369913 \\
\hline $\mathrm{H}$ & 6.345492 & -2.314139 & 0.249671 \\
\hline $\mathrm{H}$ & 6.020821 & -3.291371 & -1.209460 \\
\hline
\end{tabular}


Sum of Energies $=-1420.738476$

\section{Pink Pathway (Diels-Alder Product)}

\begin{tabular}{|c|c|c|c|}
\hline $\mathrm{C}$ & -1.429444 & -0.167318 & 0.527629 \\
\hline $\mathrm{C}$ & -1.050475 & -0.828412 & -0.670974 \\
\hline 0 & 0.151858 & -0.632470 & -1.291151 \\
\hline $\mathrm{C}$ & 0.965010 & 0.479737 & -0.866199 \\
\hline $\mathrm{C}$ & 0.949187 & 0.597837 & 0.682914 \\
\hline $\mathrm{C}$ & -0.483951 & 0.761515 & 1.183128 \\
\hline 0 & -0.754943 & 1.550507 & 2.076913 \\
\hline $\mathrm{C}$ & 1.890500 & 1.746464 & 1.012820 \\
\hline $\mathrm{C}$ & 3.152985 & 1.625506 & 0.560141 \\
\hline $\mathrm{C}$ & 3.383099 & 0.250783 & -0.021868 \\
\hline $\mathrm{C}$ & 2.432863 & 0.135017 & -1.234524 \\
\hline $\mathrm{C}$ & 1.507082 & -0.808284 & 1.243877 \\
\hline $\mathrm{C}$ & 3.019122 & -0.734353 & 1.101992 \\
\hline 0 & 4.637121 & -0.396305 & -0.240694 \\
\hline $\mathrm{C}$ & 4.150807 & -1.613941 & 0.478412 \\
\hline $\mathrm{C}$ & 3.742257 & -2.715255 & -0.493360 \\
\hline $\mathrm{C}$ & 5.221234 & -2.086968 & 1.449561 \\
\hline $\mathrm{C}$ & 0.561683 & 1.736135 & -1.696376 \\
\hline $\mathrm{C}$ & -0.873652 & 2.178394 & -1.603649 \\
\hline $\mathrm{C}$ & -1.361043 & 3.285965 & -1.020300 \\
\hline $\mathrm{C}$ & -2.844791 & 3.564769 & -1.033896 \\
\hline $\mathrm{C}$ & -0.542715 & 4.311402 & -0.276175 \\
\hline 0 & 2.780307 & -0.149188 & -2.356882 \\
\hline $\mathrm{H}$ & 1.503719 & 2.611980 & 1.538941 \\
\hline $\mathrm{H}$ & 3.940748 & 2.364891 & 0.669137 \\
\hline $\mathrm{H}$ & 3.435200 & -0.317206 & 2.022697 \\
\hline $\mathrm{H}$ & 4.606362 & -3.011741 & -1.097425 \\
\hline $\mathrm{H}$ & 3.395620 & -3.593471 & 0.064672 \\
\hline $\mathrm{H}$ & 2.949296 & -2.406692 & -1.176308 \\
\hline $\mathrm{H}$ & 4.843359 & -2.907894 & 2.070678 \\
\hline $\mathrm{H}$ & 6.097285 & -2.449480 & 0.900142 \\
\hline $\mathrm{H}$ & 5.541457 & -1.270080 & 2.103378 \\
\hline $\mathrm{H}$ & 1.245697 & 2.546851 & -1.437099 \\
\hline $\mathrm{H}$ & 0.787347 & 1.452873 & -2.732224 \\
\hline $\mathrm{H}$ & -1.588017 & 1.516685 & -2.091182 \\
\hline $\mathrm{H}$ & -3.237070 & 3.620085 & -0.008764 \\
\hline $\mathrm{H}$ & -3.063163 & 4.533015 & -1.505901 \\
\hline $\mathrm{H}$ & -3.401866 & 2.789955 & -1.569792 \\
\hline $\mathrm{H}$ & -0.808531 & 5.327686 & -0.595998 \\
\hline $\mathrm{H}$ & -0.754137 & 4.247804 & 0.800084 \\
\hline $\mathrm{H}$ & 0.534797 & 4.187723 & -0.408887 \\
\hline $\mathrm{H}$ & 1.195061 & -0.931498 & 2.284336 \\
\hline $\mathrm{H}$ & 1.059517 & -1.614455 & 0.658727 \\
\hline
\end{tabular}




$\begin{array}{lrrr}\mathrm{C} & -2.719787 & -0.464128 & 1.046635 \\ \mathrm{C} & -1.884438 & -1.740103 & -1.308303 \\ \mathrm{H} & -1.569019 & -2.217719 & -2.227821 \\ \mathrm{C} & -3.138254 & -2.015181 & -0.761137 \\ \mathrm{C} & -3.566439 & -1.381558 & 0.414651 \\ \mathrm{H} & -4.538929 & -1.599086 & 0.829844 \\ \mathrm{O} & -3.065392 & 0.169455 & 2.190112 \\ \mathrm{O} & -3.888550 & -2.914812 & -1.448950 \\ \mathrm{C} & -5.187385 & -3.237360 & -0.972563 \\ \mathrm{H} & -5.838016 & -2.353926 & -0.947412 \\ \mathrm{H} & -5.590742 & -3.962225 & -1.681498 \\ \mathrm{H} & -5.149055 & -3.690256 & 0.026409 \\ \mathrm{C} & -4.320368 & -0.111721 & 2.789370 \\ \mathrm{H} & -4.408632 & -1.170332 & 3.066560 \\ \mathrm{H} & -4.356745 & 0.502889 & 3.690064 \\ \mathrm{H} & -5.153289 & 0.162477 & 2.129015\end{array}$

Sum of Energies $=-1420.739575$

\section{Pink Pathway (Diels-Alder TS)}

$\begin{array}{lrrr}\text { C } & -1.513835 & -0.183443 & 0.571123 \\ \mathrm{C} & -1.139594 & -0.804461 & -0.647992 \\ \mathrm{O} & 0.072060 & -0.619239 & -1.247631 \\ \mathrm{C} & 0.900164 & 0.475653 & -0.828239 \\ \mathrm{C} & 0.791707 & 0.765659 & 0.645605 \\ \mathrm{C} & -0.549991 & 0.682285 & 1.284747 \\ \mathrm{O} & -0.763680 & 1.248272 & 2.354648 \\ \mathrm{C} & 1.775542 & 1.628640 & 1.165411 \\ \mathrm{C} & 3.086807 & 1.496417 & 0.725031 \\ \mathrm{C} & 3.347641 & 0.344931 & -0.073518 \\ \mathrm{C} & 2.350810 & 0.095672 & -1.179052 \\ \mathrm{C} & 1.723016 & -1.411176 & 1.180100 \\ \mathrm{C} & 3.072868 & -1.030454 & 1.169398 \\ \mathrm{O} & 4.615057 & -0.203210 & -0.281156 \\ \mathrm{C} & 4.333210 & -1.492441 & 0.379834 \\ \mathrm{C} & 4.114266 & -2.607680 & -0.638063 \\ \mathrm{C} & 5.499654 & -1.809883 & 1.310093 \\ \mathrm{C} & 0.564627 & 1.721748 & -1.728707 \\ \mathrm{C} & -0.837217 & 2.249831 & -1.612089 \\ \mathrm{C} & -1.241271 & 3.399854 & -1.046959 \\ \mathrm{C} & -2.704495 & 3.772878 & -1.038834 \\ \mathrm{C} & -0.340770 & 4.402688 & -0.369291 \\ \mathrm{O} & 2.658654 & -0.329587 & -2.273363 \\ \mathrm{H} & 1.524045 & 2.250668 & 2.019286 \\ \mathrm{H} & 3.913344 & 2.023608 & 1.193053 \\ \mathrm{H} & 3.388598 & -0.561913 & 2.101601\end{array}$




$\begin{array}{lrrr}\mathrm{H} & 5.009029 & -2.707429 & -1.260964 \\ \mathrm{H} & 3.945873 & -3.556381 & -0.115750 \\ \mathrm{H} & 3.268947 & -2.414678 & -1.297911 \\ \mathrm{H} & 5.290823 & -2.714671 & 1.892118 \\ \mathrm{H} & 6.410432 & -1.976003 & 0.724761 \\ \mathrm{H} & 5.683049 & -0.980991 & 2.000771 \\ \mathrm{H} & 1.305171 & 2.495175 & -1.507757 \\ \mathrm{H} & 0.751000 & 1.378185 & -2.753767 \\ \mathrm{H} & -1.602465 & 1.616079 & -2.057920 \\ \mathrm{H} & -3.072814 & 3.881710 & -0.009209 \\ \mathrm{H} & -2.869660 & 4.740049 & -1.534198 \\ \mathrm{H} & -3.321009 & 3.022029 & -1.542574 \\ \mathrm{H} & -0.476643 & 5.401614 & -0.806288 \\ \mathrm{H} & -0.601715 & 4.486743 & 0.694473 \\ \mathrm{H} & 0.719625 & 4.148004 & -0.427952 \\ \mathrm{H} & 1.177691 & -1.425306 & 2.116213 \\ \mathrm{H} & 1.288802 & -1.979063 & 0.363926 \\ \mathrm{C} & -2.816297 & -0.478213 & 1.065132 \\ \mathrm{C} & -1.979076 & -1.683708 & -1.324706 \\ \mathrm{H} & -1.657499 & -2.137032 & -2.254325 \\ \mathrm{C} & -3.241343 & -1.959133 & -0.800716 \\ \mathrm{C} & -3.671543 & -1.356536 & 0.390216 \\ \mathrm{H} & -4.653931 & -1.567259 & 0.785002 \\ \mathrm{O} & -3.176413 & 0.130242 & 2.217405 \\ \mathrm{O} & -3.998355 & -2.825023 & -1.525117 \\ \mathrm{C} & -5.303650 & -3.147350 & -1.067812 \\ \mathrm{H} & -5.710383 & -3.845282 & -1.801535 \\ \mathrm{H} & -5.277702 & -3.631285 & -0.083060 \\ \mathrm{H} & -5.945018 & -2.258012 & -1.019446 \\ \mathrm{C} & -4.455144 & -0.130484 & 2.772453 \\ \mathrm{H} & -4.579803 & -1.192358 & 3.022110 \\ \mathrm{H} & -4.501359 & 0.463366 & 3.686598 \\ \mathrm{H} & -5.261524 & 0.181009 & 2.095471\end{array}$

Sum of Energies $=-1420.695430$

\section{Green Pathway (Claisen Intermediate)}

$\begin{array}{lrrr}\text { C } & -2.297379 & -1.224946 & 0.151899 \\ C & -2.231757 & 0.142265 & -0.198367 \\ \text { O } & -1.054916 & 0.843573 & -0.250475 \\ C & 0.140086 & 0.276407 & 0.068816 \\ C & 0.192288 & -1.135035 & 0.422276 \\ C & -1.063325 & -1.954162 & 0.485650 \\ \text { O } & -1.007377 & -3.138660 & 0.796469 \\ C & 1.382060 & -1.707180 & 0.697872 \\ C & 2.674294 & -0.971622 & 0.597150\end{array}$




\begin{tabular}{|c|c|c|c|}
\hline $\mathrm{C}$ & 2.543232 & 0.548512 & 0.463245 \\
\hline $\mathrm{C}$ & 1.242351 & 1.089769 & 0.040992 \\
\hline $\mathrm{O}$ & 1.148448 & 2.367636 & -0.419261 \\
\hline $\mathrm{C}$ & 1.096167 & 3.534882 & 0.464510 \\
\hline C & 2.433508 & 4.277940 & 0.311056 \\
\hline $\mathrm{C}$ & 0.844416 & 3.142994 & 1.925996 \\
\hline $\mathrm{C}$ & -0.027976 & 4.421722 & -0.034610 \\
\hline C & -0.812549 & 4.192577 & -1.085769 \\
\hline $\mathrm{O}$ & 3.512328 & 1.274187 & 0.676954 \\
\hline C & 3.481094 & -1.550883 & -0.636586 \\
\hline $\mathrm{C}$ & 4.919934 & -1.119288 & -0.678141 \\
\hline C & 6.008481 & -1.905910 & -0.682759 \\
\hline $\mathrm{C}$ & 6.002665 & -3.414947 & -0.641575 \\
\hline C & 7.388826 & -1.294318 & -0.742633 \\
\hline $\mathrm{H}$ & 1.393363 & -2.764777 & 0.948836 \\
\hline $\mathrm{H}$ & 3.286988 & -1.166850 & 1.486267 \\
\hline $\mathrm{H}$ & 2.423856 & 5.212155 & 0.885115 \\
\hline $\mathrm{H}$ & 3.251650 & 3.643231 & 0.656698 \\
\hline $\mathrm{H}$ & 2.597842 & 4.523360 & -0.742582 \\
\hline $\mathrm{H}$ & -0.085752 & 2.575442 & 2.028062 \\
\hline $\mathrm{H}$ & 1.677505 & 2.555119 & 2.322345 \\
\hline $\mathrm{H}$ & 0.753359 & 4.049849 & 2.533437 \\
\hline $\mathrm{H}$ & -0.145946 & 5.341928 & 0.539228 \\
\hline $\mathrm{H}$ & -1.576007 & 4.907593 & -1.381457 \\
\hline $\mathrm{H}$ & -0.709121 & 3.291409 & -1.679725 \\
\hline $\mathrm{H}$ & 3.392587 & -2.640397 & -0.599994 \\
\hline $\mathrm{H}$ & 2.957541 & -1.227564 & -1.547092 \\
\hline $\mathrm{H}$ & 5.072447 & -0.043018 & -0.700662 \\
\hline $\mathrm{H}$ & 6.485182 & -3.827776 & -1.538543 \\
\hline $\mathrm{H}$ & 6.584987 & -3.778649 & 0.216435 \\
\hline $\mathrm{H}$ & 5.002841 & -3.850122 & -0.573647 \\
\hline $\mathrm{H}$ & 7.991783 & -1.595428 & 0.125802 \\
\hline $\mathrm{H}$ & 7.935366 & -1.636538 & -1.633023 \\
\hline $\mathrm{H}$ & 7.349458 & -0.201237 & -0.766119 \\
\hline $\mathrm{C}$ & -3.593443 & -1.817893 & 0.158092 \\
\hline $\mathrm{C}$ & -3.349027 & 0.898757 & -0.518901 \\
\hline $\mathrm{H}$ & -3.247070 & 1.946778 & -0.771477 \\
\hline C & -4.732058 & -1.071543 & -0.164857 \\
\hline $\mathrm{H}$ & -5.705227 & -1.538862 & -0.153289 \\
\hline C & -4.602534 & 0.284178 & -0.500542 \\
\hline $\mathrm{O}$ & -3.656418 & -3.123216 & 0.489507 \\
\hline $\mathrm{O}$ & -5.648633 & 1.084428 & -0.823626 \\
\hline C & -4.916170 & -3.778409 & 0.512303 \\
\hline $\mathrm{H}$ & -4.701244 & -4.808121 & 0.800772 \\
\hline $\mathrm{H}$ & -5.591992 & -3.326645 & 1.249728 \\
\hline $\mathrm{H}$ & -5.393010 & -3.768436 & -0.476155 \\
\hline $\mathrm{C}$ & -6.961004 & 0.537791 & -0.828170 \\
\hline $\mathrm{H}$ & -7.621194 & 1.358150 & -1.113489 \\
\hline
\end{tabular}




$\begin{array}{rrrr}\mathrm{H} & -7.054423 & -0.274876 & -1.559403 \\ \mathrm{H} & -7.246300 & 0.170748 & 0.165709\end{array}$

Sum of Energies $=-1420.773320$

\section{Green Pathway (Claisen TS)}

\begin{tabular}{|c|c|c|c|}
\hline $\mathrm{C}$ & -2.475248 & -0.935532 & 0.147900 \\
\hline C & -2.004381 & 0.368841 & -0.121423 \\
\hline $\mathrm{O}$ & -0.680365 & 0.703617 & -0.068596 \\
\hline $\mathrm{C}$ & 0.270928 & -0.222490 & 0.264491 \\
\hline $\mathrm{C}$ & -0.096363 & -1.567067 & 0.550557 \\
\hline $\mathrm{C}$ & -1.517288 & -2.000974 & 0.504792 \\
\hline $\mathrm{O}$ & -1.823054 & -3.165632 & 0.750640 \\
\hline $\mathrm{C}$ & 0.893713 & -2.466478 & 0.905300 \\
\hline $\mathrm{C}$ & 2.248628 & -2.082009 & 0.911938 \\
\hline $\mathrm{C}$ & 2.635463 & -0.695454 & 0.700173 \\
\hline $\mathrm{C}$ & 1.583976 & 0.222261 & 0.306184 \\
\hline $\mathrm{O}$ & 1.884750 & 1.502628 & -0.070368 \\
\hline $\mathrm{C}$ & 2.025674 & 2.583419 & 0.903473 \\
\hline $\mathrm{C}$ & 3.529124 & 2.845766 & 1.098142 \\
\hline $\mathrm{C}$ & 1.352680 & 2.252577 & 2.243398 \\
\hline $\mathrm{C}$ & 1.374821 & 3.809924 & 0.295899 \\
\hline $\mathrm{C}$ & 0.710509 & 3.864083 & -0.856853 \\
\hline 0 & 3.852909 & -0.347668 & 0.763813 \\
\hline $\mathrm{C}$ & 4.840018 & -1.057603 & -1.220052 \\
\hline $\mathrm{C}$ & 6.255799 & -0.719176 & -0.855718 \\
\hline $\mathrm{C}$ & 4.126775 & -0.038112 & -2.060464 \\
\hline $\mathrm{C}$ & 4.369764 & -2.354270 & -1.026502 \\
\hline $\mathrm{C}$ & 3.039165 & -2.740384 & -1.143338 \\
\hline $\mathrm{H}$ & 0.590824 & -3.478860 & 1.154093 \\
\hline $\mathrm{H}$ & 2.988408 & -2.718438 & 1.384663 \\
\hline $\mathrm{H}$ & 3.688258 & 3.692104 & 1.777157 \\
\hline $\mathrm{H}$ & 4.016413 & 1.955656 & 1.501407 \\
\hline $\mathrm{H}$ & 3.989784 & 3.087762 & 0.134984 \\
\hline $\mathrm{H}$ & 0.288820 & 2.041137 & 2.105905 \\
\hline $\mathrm{H}$ & 1.831414 & 1.394754 & 2.726290 \\
\hline $\mathrm{H}$ & 1.444524 & 3.111815 & 2.916480 \\
\hline $\mathrm{H}$ & 1.502626 & 4.715005 & 0.891252 \\
\hline $\mathrm{H}$ & 0.288631 & 4.798618 & -1.217606 \\
\hline $\mathrm{H}$ & 0.572031 & 2.979516 & -1.468510 \\
\hline $\mathrm{H}$ & 6.847404 & -0.506946 & -1.757858 \\
\hline $\mathrm{H}$ & 6.266767 & 0.185428 & -0.238903 \\
\hline $\mathrm{H}$ & 6.743583 & -1.524539 & -0.299970 \\
\hline $\mathrm{H}$ & 3.043879 & -0.169684 & -2.076692 \\
\hline $\mathrm{H}$ & 4.333858 & 0.972503 & -1.703329 \\
\hline $\mathrm{H}$ & 4.490896 & -0.114167 & -3.096180 \\
\hline
\end{tabular}




$\begin{array}{lrrr}\mathrm{H} & 5.047313 & -3.051532 & -0.536252 \\ \mathrm{H} & 2.763156 & -3.777317 & -0.985664 \\ \mathrm{H} & 2.316816 & -2.152499 & -1.697429 \\ \mathrm{C} & -2.841972 & 1.425477 & -0.460236 \\ \mathrm{H} & -2.431142 & 2.409349 & -0.650303 \\ \mathrm{C} & -3.883270 & -1.133407 & 0.052533 \\ \mathrm{C} & -4.741088 & -0.082769 & -0.288240 \\ \mathrm{H} & -5.804984 & -0.253975 & -0.354424 \\ \mathrm{C} & -4.213114 & 1.192945 & -0.542059 \\ \mathrm{O} & -4.335976 & -2.379896 & 0.308000 \\ \mathrm{O} & -4.971854 & 2.269903 & -0.876921 \\ \mathrm{C} & -5.727602 & -2.643807 & 0.231751 \\ \mathrm{H} & -6.118840 & -2.462374 & -0.777963 \\ \mathrm{H} & -5.836143 & -3.701240 & 0.477247 \\ \mathrm{H} & -6.293683 & -2.042259 & 0.955040 \\ \mathrm{C} & -6.380320 & 2.115905 & -0.974751 \\ \mathrm{H} & -6.653553 & 1.387610 & -1.749051 \\ \mathrm{H} & -6.819961 & 1.810805 & -0.016509 \\ \mathrm{H} & -6.766715 & 3.098144 & -1.251801\end{array}$

Sum of Energies $=-1420.742847$

\section{Green Pathway (Diels-Alder Product)}

$\begin{array}{lrrr}\text { C } & -1.807188 & 0.341692 & -0.309361 \\ \mathrm{C} & -1.811475 & -0.896669 & 0.374521 \\ \mathrm{O} & -0.654204 & -1.603141 & 0.617398 \\ \mathrm{C} & 0.508857 & -1.059347 & 0.208400 \\ \mathrm{C} & 0.635940 & 0.122167 & -0.429185 \\ \mathrm{C} & -0.529691 & 0.928547 & -0.794027 \\ \mathrm{O} & -0.423875 & 1.971446 & -1.439999 \\ \mathrm{C} & 2.072208 & 0.420809 & -0.782065 \\ \mathrm{C} & 2.898972 & 0.430092 & 0.549731 \\ \mathrm{C} & 2.611357 & -0.862807 & 1.376088 \\ \mathrm{C} & 1.790996 & -1.801284 & 0.485456 \\ \mathrm{C} & 3.231389 & 2.934692 & 0.777709 \\ \mathrm{C} & 2.576608 & 4.048253 & 0.411546 \\ \mathrm{C} & 1.096071 & 4.281442 & 0.592950 \\ \mathrm{C} & 3.313782 & 5.188357 & -0.251195 \\ \mathrm{C} & 2.676249 & 1.691618 & 1.418157 \\ \mathrm{O} & 3.042721 & -1.085970 & 2.482992 \\ \mathrm{O} & 1.663954 & -3.211312 & 0.637333 \\ \mathrm{C} & 2.103617 & -3.420092 & -0.772099 \\ \mathrm{C} & 2.543537 & -0.755991 & -1.722340 \\ \mathrm{C} & 2.667759 & -1.961756 & -0.788032 \\ \mathrm{C} & 0.909790 & -3.705445 & -1.674993 \\ \mathrm{C} & 3.141467 & -4.529548 & -0.824635 \\ & & & \end{array}$




$\begin{array}{lrrr}\mathrm{H} & 2.150549 & 1.387024 & -1.284278 \\ \mathrm{H} & 3.972094 & 0.384399 & 0.307272 \\ \mathrm{H} & 4.305417 & 2.896403 & 0.580943 \\ \mathrm{H} & 0.624745 & 3.556978 & 1.260692 \\ \mathrm{H} & 0.914255 & 5.283821 & 1.003206 \\ \mathrm{H} & 0.571650 & 4.220515 & -0.369109 \\ \mathrm{H} & 4.375914 & 4.964177 & -0.393672 \\ \mathrm{H} & 2.878538 & 5.417642 & -1.234053 \\ \mathrm{H} & 3.236338 & 6.109067 & 0.344177 \\ \mathrm{H} & 3.176661 & 1.505066 & 2.377977 \\ \mathrm{H} & 1.608134 & 1.792881 & 1.634945 \\ \mathrm{H} & 3.506537 & -0.523157 & -2.189742 \\ \mathrm{H} & 3.707418 & -2.037731 & -0.451823 \\ \mathrm{H} & 1.239081 & -3.821486 & -2.714331 \\ \mathrm{H} & 0.424285 & -4.635768 & -1.361888 \\ \mathrm{H} & 0.164256 & -2.907383 & -1.640654 \\ \mathrm{H} & 3.955710 & -4.331235 & -0.121250 \\ \mathrm{H} & 2.686288 & -5.489847 & -0.557731 \\ \mathrm{H} & 3.559539 & -4.617426 & -1.834603 \\ \mathrm{H} & 1.810407 & -0.887577 & -2.523853 \\ \mathrm{C} & -3.079390 & 0.958555 & -0.489418 \\ \mathrm{C} & -4.249572 & 0.358354 & -0.015276 \\ \mathrm{H} & -5.202043 & 0.845315 & -0.162164 \\ \mathrm{C} & -2.963243 & -1.510792 & 0.851731 \\ \mathrm{H} & -2.906430 & -2.460755 & 1.368518 \\ \mathrm{C} & -4.186117 & -0.875492 & 0.652658 \\ \mathrm{O} & -3.085557 & 2.145458 & -1.134375 \\ \mathrm{O} & -5.274253 & -1.526245 & 1.141607 \\ \mathrm{C} & -6.557860 & -0.939587 & 0.979046 \\ \mathrm{H} & -6.813672 & -0.818116 & -0.081310 \\ \mathrm{H} & -7.260799 & -1.633826 & 1.442203 \\ \mathrm{H} & -6.623240 & 0.032764 & 1.483740 \\ \mathrm{C} & -4.317495 & 2.817453 & -1.342166 \\ \mathrm{H} & -5.004891 & 2.220567 & -1.955950 \\ \mathrm{H} & -4.804952 & 3.071759 & -0.391777 \\ \mathrm{H} & -4.061485 & 3.734704 & -1.874330\end{array}$

Sum of Energies $=-1420.778461$

\section{Green Pathway (Diels-Alder TS)}

$\begin{array}{rrrr}\mathrm{C} & 1.832432 & 0.520702 & 0.220687 \\ \mathrm{C} & 1.874317 & -0.681257 & -0.522962 \\ \mathrm{O} & 0.747905 & -1.398593 & -0.826945 \\ \mathrm{C} & -0.482757 & -0.902840 & -0.510689 \\ \mathrm{C} & -0.640164 & 0.286060 & 0.216501 \\ \mathrm{C} & 0.539233 & 1.034848 & 0.712948\end{array}$




\begin{tabular}{|c|c|c|c|}
\hline $\mathrm{O}$ & 0.400499 & 1.997059 & 1.466829 \\
\hline $\mathrm{C}$ & -1.934829 & 0.556385 & 0.683662 \\
\hline C & -3.082307 & 0.234602 & -0.243377 \\
\hline C & -2.898730 & -1.116005 & -0.962518 \\
\hline C & -1.592310 & -1.749222 & -0.686208 \\
\hline $\mathrm{C}$ & -3.755109 & 2.660976 & -0.702020 \\
\hline $\mathrm{C}$ & -3.054373 & 3.790317 & -0.509543 \\
\hline C & -1.603951 & 3.977957 & -0.886440 \\
\hline C & -3.692224 & 4.988377 & 0.152989 \\
\hline $\mathrm{C}$ & -3.281564 & 1.367798 & -1.307171 \\
\hline 0 & -3.782352 & -1.616585 & -1.642667 \\
\hline $\mathrm{O}$ & -1.420343 & -3.123676 & -0.734051 \\
\hline C & -1.607376 & -3.444740 & 0.692713 \\
\hline C & -2.096742 & -1.184353 & 2.085980 \\
\hline $\mathrm{C}$ & -2.412526 & -2.225620 & 1.219112 \\
\hline C & -0.249669 & -3.630982 & 1.366298 \\
\hline $\mathrm{C}$ & -2.443944 & -4.720682 & 0.755388 \\
\hline $\mathrm{H}$ & -2.045551 & 1.412230 & 1.342686 \\
\hline $\mathrm{H}$ & -4.021457 & 0.156911 & 0.317613 \\
\hline $\mathrm{H}$ & -4.791467 & 2.646400 & -0.358747 \\
\hline $\mathrm{H}$ & -1.238151 & 3.215645 & -1.578765 \\
\hline $\mathrm{H}$ & -1.449538 & 4.959030 & -1.354287 \\
\hline $\mathrm{H}$ & -0.964047 & 3.939786 & 0.005819 \\
\hline $\mathrm{H}$ & -4.729363 & 4.793346 & 0.444127 \\
\hline $\mathrm{H}$ & -3.134050 & 5.282339 & 1.053009 \\
\hline $\mathrm{H}$ & -3.683546 & 5.860713 & -0.515555 \\
\hline $\mathrm{H}$ & -4.030654 & 0.984308 & -2.011390 \\
\hline $\mathrm{H}$ & -2.349292 & 1.496228 & -1.865131 \\
\hline $\mathrm{H}$ & -2.897070 & -0.717891 & 2.654016 \\
\hline $\mathrm{H}$ & -3.472406 & -2.336391 & 0.995131 \\
\hline $\mathrm{H}$ & -0.385448 & -3.934123 & 2.410670 \\
\hline $\mathrm{H}$ & 0.313289 & -4.411626 & 0.845584 \\
\hline $\mathrm{H}$ & 0.348878 & -2.717389 & 1.352482 \\
\hline $\mathrm{H}$ & -3.379740 & -4.585655 & 0.205153 \\
\hline $\mathrm{H}$ & -1.896708 & -5.554287 & 0.302884 \\
\hline $\mathrm{H}$ & -2.674257 & -4.978889 & 1.795335 \\
\hline $\mathrm{H}$ & -1.111691 & -1.098225 & 2.533850 \\
\hline $\mathrm{C}$ & 3.083840 & 1.158985 & 0.462886 \\
\hline C & 3.052966 & -1.243427 & -0.997645 \\
\hline $\mathrm{H}$ & 3.032991 & -2.168640 & -1.559955 \\
\hline C & 4.279283 & 0.609286 & -0.009102 \\
\hline $\mathrm{H}$ & 5.216513 & 1.109456 & 0.183632 \\
\hline $\mathrm{C}$ & 4.257054 & -0.592808 & -0.734870 \\
\hline $\mathrm{O}$ & 3.043961 & 2.314961 & 1.158473 \\
\hline O & 5.369295 & -1.198280 & -1.224116 \\
\hline $\mathrm{C}$ & 4.252676 & 3.010995 & 1.421455 \\
\hline $\mathrm{H}$ & 4.752280 & 3.317461 & 0.493267 \\
\hline $\mathrm{H}$ & 3.959856 & 3.897618 & 1.985436 \\
\hline
\end{tabular}




$\begin{array}{rrrr}\mathrm{H} & 4.942439 & 2.406999 & 2.025147 \\ \mathrm{C} & 6.638260 & -0.601680 & -0.992730 \\ \mathrm{H} & 7.365881 & -1.262704 & -1.466070 \\ \mathrm{H} & 6.703525 & 0.394592 & -1.448069 \\ \mathrm{H} & 6.858148 & -0.528380 & 0.079870\end{array}$

Sum of Energies $=-1420.716834$

1-O-Methyllateriflorone System

13

\begin{tabular}{|c|c|c|c|}
\hline $\mathrm{C}$ & -2.285425 & -3.829922 & -1.051447 \\
\hline 0 & -2.247769 & -2.451578 & -0.727364 \\
\hline $\mathrm{C}$ & -1.033270 & -1.896576 & -0.425325 \\
\hline $\mathrm{C}$ & 0.162540 & -2.603839 & -0.359058 \\
\hline $\mathrm{C}$ & 1.357475 & -1.952034 & -0.029499 \\
\hline $\mathrm{C}$ & 1.362909 & -0.575106 & 0.185054 \\
\hline $\mathrm{C}$ & 0.160917 & 0.168473 & 0.091989 \\
\hline $\mathrm{C}$ & -1.047438 & -0.508539 & -0.143598 \\
\hline $\mathrm{O}$ & -2.231328 & 0.176573 & -0.217441 \\
\hline $\mathrm{C}$ & -3.121464 & 0.281440 & 0.939245 \\
\hline $\mathrm{C}$ & -2.903276 & 1.663297 & 1.580076 \\
\hline $\mathrm{C}$ & -2.886596 & -0.824725 & 1.979734 \\
\hline C & -4.536912 & 0.188846 & 0.406768 \\
\hline $\mathrm{C}$ & -4.890909 & -0.119434 & -0.838809 \\
\hline 0 & 0.159227 & 1.509996 & 0.379482 \\
\hline $\mathrm{C}$ & 0.338371 & 2.515838 & -0.685782 \\
\hline $\mathrm{C}$ & -0.649893 & 2.287418 & -1.841283 \\
\hline C & 1.775672 & 2.495147 & -1.208921 \\
\hline C & -0.017284 & 3.803408 & 0.028956 \\
\hline $\mathrm{C}$ & 0.688912 & 4.932702 & 0.048897 \\
\hline C & 2.648521 & -2.715895 & 0.136213 \\
\hline $\mathrm{O}$ & 3.618400 & -1.946166 & 0.840219 \\
\hline $\mathrm{C}$ & 3.746589 & -0.614927 & 0.381930 \\
\hline C & 4.191249 & -0.545741 & -1.084540 \\
\hline $\mathrm{C}$ & 4.728526 & 0.062917 & 1.324847 \\
\hline $\mathrm{O}$ & 2.503100 & 0.098947 & 0.538275 \\
\hline $\mathrm{H}$ & -3.327697 & -4.051857 & -1.288130 \\
\hline $\mathrm{H}$ & -3.593725 & 1.809321 & 2.419247 \\
\hline $\mathrm{H}$ & -1.877354 & 1.757151 & 1.944461 \\
\hline $\mathrm{H}$ & -3.087823 & 2.452705 & 0.844961 \\
\hline $\mathrm{H}$ & -3.029331 & -1.812094 & 1.536737 \\
\hline $\mathrm{H}$ & -1.880904 & -0.759574 & 2.407643 \\
\hline $\mathrm{H}$ & -3.607028 & -0.704892 & 2.796116 \\
\hline $\mathrm{H}$ & -5.302498 & 0.408727 & 1.151944 \\
\hline
\end{tabular}




$\begin{array}{rrrr}\mathrm{H} & -5.937061 & -0.157053 & -1.131324 \\ \mathrm{H} & -4.146996 & -0.346244 & -1.594201 \\ \mathrm{H} & -0.520831 & 3.078722 & -2.587437 \\ \mathrm{H} & -0.463733 & 1.324136 & -2.328165 \\ \mathrm{H} & -1.682936 & 2.292837 & -1.490716 \\ \mathrm{H} & 2.488873 & 2.628584 & -0.393421 \\ \mathrm{H} & 1.986184 & 1.543342 & -1.703190 \\ \mathrm{H} & 1.917170 & 3.291806 & -1.947365 \\ \mathrm{H} & -0.972776 & 3.760411 & 0.548590 \\ \mathrm{H} & 0.321828 & 5.811607 & 0.572075 \\ \mathrm{H} & 1.650193 & 5.035859 & -0.446130 \\ \mathrm{H} & -1.657791 & -4.057037 & -1.924019 \\ \mathrm{H} & -1.964891 & -4.456449 & -0.207600 \\ \mathrm{H} & 0.181777 & -3.673913 & -0.537679 \\ \mathrm{H} & 2.494043 & -3.612028 & 0.746153 \\ \mathrm{H} & 3.049275 & -3.049396 & -0.835273 \\ \mathrm{H} & 4.398301 & 0.491276 & -1.362635 \\ \mathrm{H} & 5.101325 & -1.137886 & -1.224495 \\ \mathrm{H} & 3.418110 & -0.926583 & -1.758106 \\ \mathrm{H} & 4.808600 & 1.126664 & 1.085268 \\ \mathrm{H} & 5.714814 & -0.401372 & 1.235650 \\ \mathrm{H} & 4.374562 & -0.045431 & 2.352994\end{array}$

Sum of Energies $=-1194.330075$

14

$\begin{array}{lrrr}\mathrm{C} & 4.553269 & 0.787077 & -1.452702 \\ \mathrm{O} & 3.354917 & 1.305890 & -2.040033 \\ \mathrm{C} & 2.186390 & 1.128062 & -1.349738 \\ \mathrm{C} & 1.380207 & 2.199058 & -1.126985 \\ \mathrm{C} & 0.076467 & 2.109695 & -0.519496 \\ \mathrm{C} & -0.401268 & 0.913394 & -0.099760 \\ \mathrm{C} & 0.341959 & -0.402477 & -0.287583 \\ \mathrm{C} & 1.726680 & -0.223891 & -1.013493 \\ \mathrm{O} & 2.355379 & -1.220675 & -1.358011 \\ \mathrm{O} & 0.427766 & -0.892825 & 1.065482 \\ \mathrm{C} & 1.383538 & -1.838333 & 1.605987 \\ \mathrm{C} & 1.457052 & -3.170792 & 0.841829 \\ \mathrm{C} & 0.799915 & -2.101738 & 3.010496 \\ \mathrm{C} & 2.759073 & -1.209220 & 1.777212 \\ \mathrm{C} & 2.986027 & 0.087593 & 1.986462 \\ \mathrm{C} & -0.475956 & -1.361750 & -1.212626 \\ \mathrm{C} & -1.777664 & -1.853533 & -0.643934 \\ \mathrm{C} & -2.922266 & -2.085431 & -1.305008 \\ \mathrm{C} & -3.135015 & -1.851751 & -2.780891 \\ \mathrm{C} & -4.128357 & -2.635674 & -0.580355\end{array}$




$\begin{array}{lrrr}\mathrm{C} & -2.229803 & 1.998215 & 0.979350 \\ \mathrm{O} & -1.584491 & 0.774357 & 0.533974 \\ \mathrm{C} & -3.709467 & 1.666302 & 1.077001 \\ \mathrm{C} & -1.614201 & 2.398705 & 2.322998 \\ \mathrm{H} & 5.360118 & 1.077598 & -2.129486 \\ \mathrm{H} & 2.058173 & -3.883080 & 1.419596 \\ \mathrm{H} & 0.454313 & -3.595000 & 0.728644 \\ \mathrm{H} & 1.913065 & -3.049269 & -0.140313 \\ \mathrm{H} & 0.669747 & -1.156744 & 3.544795 \\ \mathrm{H} & -0.171757 & -2.600879 & 2.929366 \\ \mathrm{H} & 1.474566 & -2.739612 & 3.591290 \\ \mathrm{H} & 3.594047 & -1.909686 & 1.780037 \\ \mathrm{H} & 3.989831 & 0.468700 & 2.152876 \\ \mathrm{H} & 2.176640 & 0.812663 & 1.992365 \\ \mathrm{H} & -0.619572 & -0.847881 & -2.170666 \\ \mathrm{H} & 0.192249 & -2.204489 & -1.424233 \\ \mathrm{H} & -1.753558 & -2.067348 & 0.421998 \\ \mathrm{H} & -3.365735 & -2.796196 & -3.294175 \\ \mathrm{H} & -3.996931 & -1.190956 & -2.949438 \\ \mathrm{H} & -2.270372 & -1.408356 & -3.280407 \\ \mathrm{H} & -4.449593 & -3.594817 & -1.011510 \\ \mathrm{H} & -3.927566 & -2.793715 & 0.484017 \\ \mathrm{H} & -4.987715 & -1.955099 & -0.667275 \\ \mathrm{H} & 4.727334 & 1.236362 & -0.466141 \\ \mathrm{H} & 4.513093 & -0.299939 & -1.361965 \\ \mathrm{H} & 1.739453 & 3.174720 & -1.448909 \\ \mathrm{H} & -3.861448 & 0.822625 & 1.755153 \\ \mathrm{H} & -4.261718 & 2.533461 & 1.450003 \\ \mathrm{H} & -4.087043 & 1.401287 & 0.086476 \\ \mathrm{H} & -0.532425 & 2.536006 & 2.242882 \\ \mathrm{H} & -2.064364 & 3.333780 & 2.671014 \\ \mathrm{H} & -1.799025 & 1.615959 & 3.063565 \\ \mathrm{C} & -0.769113 & 3.344715 & -0.343654 \\ \mathrm{H} & -0.338289 & 4.031296 & 0.404273 \\ \mathrm{H} & -0.846676 & 3.901738 & -1.284328 \\ \mathrm{O} & -2.110277 & 3.002943 & -0.001432\end{array}$

Sum of Energies $=-1194.314924$

\section{$14 \mathrm{TS}$}

$\begin{array}{lrrr}\mathrm{C} & -2.157617 & -3.560160 & 1.990861 \\ \mathrm{O} & -2.236974 & -2.254847 & 1.451706 \\ \mathrm{C} & -1.089913 & -1.702245 & 0.956528 \\ \mathrm{C} & 0.135116 & -2.326328 & 0.913875 \\ \mathrm{C} & 1.289213 & -1.665000 & 0.410365 \\ \mathrm{C} & 1.196077 & -0.368504 & -0.044810\end{array}$




\begin{tabular}{|c|c|c|c|}
\hline C & -0.062317 & 0.315442 & -0.047424 \\
\hline C & -1.238764 & -0.312792 & 0.532162 \\
\hline $\mathrm{O}$ & -2.349850 & 0.289530 & 0.576697 \\
\hline $\mathrm{C}$ & -3.272685 & 0.116663 & -1.570017 \\
\hline C & -4.348238 & 1.151233 & -1.406481 \\
\hline $\mathrm{C}$ & -3.714721 & -1.304279 & -1.364651 \\
\hline $\mathrm{C}$ & -2.056978 & 0.468396 & -2.150706 \\
\hline $\mathrm{C}$ & -0.917216 & -0.325018 & -2.153851 \\
\hline $\mathrm{O}$ & -0.099749 & 1.644961 & -0.366638 \\
\hline $\mathrm{C}$ & 0.036514 & 2.692727 & 0.669612 \\
\hline $\mathrm{C}$ & -1.147370 & 3.634803 & 0.409195 \\
\hline C & 1.369218 & 3.390535 & 0.390385 \\
\hline $\mathrm{C}$ & -0.083042 & 2.127764 & 2.069056 \\
\hline C & 0.846827 & 2.181526 & 3.023138 \\
\hline C & 2.632977 & -2.346235 & 0.399979 \\
\hline $\mathrm{O}$ & 3.688324 & -1.412335 & 0.183061 \\
\hline $\mathrm{C}$ & 3.440084 & -0.474083 & -0.843959 \\
\hline $\mathrm{C}$ & 3.244837 & -1.140891 & -2.211562 \\
\hline $\mathrm{C}$ & 4.614784 & 0.491452 & -0.827018 \\
\hline $\mathrm{O}$ & 2.273925 & 0.320462 & -0.539785 \\
\hline $\mathrm{H}$ & -3.161388 & -3.802646 & 2.345680 \\
\hline $\mathrm{H}$ & -5.202710 & 0.934460 & -2.064342 \\
\hline $\mathrm{H}$ & -4.719383 & 1.133660 & -0.375937 \\
\hline $\mathrm{H}$ & -3.985924 & 2.158911 & -1.627338 \\
\hline $\mathrm{H}$ & -2.884200 & -2.009566 & -1.311427 \\
\hline $\mathrm{H}$ & -4.295599 & -1.396904 & -0.444194 \\
\hline $\mathrm{H}$ & -4.358600 & -1.605100 & -2.205301 \\
\hline $\mathrm{H}$ & -1.918197 & 1.517465 & -2.398453 \\
\hline $\mathrm{H}$ & -0.005878 & 0.052970 & -2.604081 \\
\hline $\mathrm{H}$ & -0.952354 & -1.395296 & -1.984914 \\
\hline $\mathrm{H}$ & -1.120564 & 4.490062 & 1.093647 \\
\hline $\mathrm{H}$ & -1.106529 & 4.009573 & -0.619589 \\
\hline $\mathrm{H}$ & -2.085292 & 3.090274 & 0.545924 \\
\hline $\mathrm{H}$ & 2.208841 & 2.707861 & 0.539376 \\
\hline $\mathrm{H}$ & 1.387138 & 3.723131 & -0.651920 \\
\hline $\mathrm{H}$ & 1.494874 & 4.266657 & 1.035893 \\
\hline $\mathrm{H}$ & -1.050583 & 1.682258 & 2.289028 \\
\hline $\mathrm{H}$ & 0.652627 & 1.780862 & 4.014717 \\
\hline $\mathrm{H}$ & 1.828585 & 2.618780 & 2.865675 \\
\hline $\mathrm{H}$ & -1.853939 & -4.296452 & 1.232695 \\
\hline $\mathrm{H}$ & -1.453030 & -3.607641 & 2.833003 \\
\hline $\mathrm{H}$ & 0.250595 & -3.342886 & 1.277916 \\
\hline $\mathrm{H}$ & 2.848606 & -2.800773 & 1.373511 \\
\hline $\mathrm{H}$ & 2.674165 & -3.155120 & -0.349494 \\
\hline $\mathrm{H}$ & 3.170021 & -0.375949 & -2.989906 \\
\hline $\mathrm{H}$ & 4.097452 & -1.790330 & -2.434519 \\
\hline $\mathrm{H}$ & 2.330781 & -1.740788 & -2.238110 \\
\hline $\mathrm{H}$ & 4.438874 & 1.310666 & -1.529421 \\
\hline
\end{tabular}




$\begin{array}{lrrr}\mathrm{H} & 5.534137 & -0.031184 & -1.106170 \\ \mathrm{H} & 4.731350 & 0.900844 & 0.179661\end{array}$

Sum of Energies $=-1194.290769$

15

\begin{tabular}{|c|c|c|c|}
\hline $\mathrm{C}$ & 4.172664 & -1.286718 & -2.069966 \\
\hline O & 3.943108 & -0.346602 & -1.022363 \\
\hline C & 2.611063 & -0.059232 & -0.682391 \\
\hline C & 1.758509 & 0.522412 & -1.791857 \\
\hline C & 0.538192 & 0.933751 & -1.427604 \\
\hline $\mathrm{C}$ & 0.171160 & 0.663893 & 0.018164 \\
\hline $\mathrm{C}$ & 1.241034 & 1.184573 & 1.061967 \\
\hline $\mathrm{C}$ & 2.644244 & 1.016806 & 0.442254 \\
\hline $\mathrm{C}$ & 1.778665 & -1.256816 & -0.164622 \\
\hline $\mathrm{C}$ & 0.345584 & -0.857743 & 0.328213 \\
\hline 0 & 2.186714 & -2.396709 & -0.109050 \\
\hline $\mathrm{C}$ & -0.688623 & -1.841062 & -0.232423 \\
\hline $\mathrm{C}$ & -2.041646 & -1.812585 & 0.429117 \\
\hline $\mathrm{C}$ & -3.227592 & -2.083253 & -0.136669 \\
\hline $\mathrm{C}$ & -3.434150 & -2.417331 & -1.594548 \\
\hline $\mathrm{C}$ & -4.491493 & -2.089030 & 0.692160 \\
\hline $\mathrm{O}$ & 0.371537 & -0.937611 & 1.751858 \\
\hline $\mathrm{C}$ & 0.954199 & 0.275968 & 2.310115 \\
\hline $\mathrm{C}$ & 2.181191 & -0.146199 & 3.128862 \\
\hline $\mathrm{C}$ & -0.084977 & 0.906310 & 3.247291 \\
\hline $\mathrm{O}$ & -1.144032 & 1.065674 & 0.373785 \\
\hline $\mathrm{C}$ & -1.828090 & 2.014502 & -0.451609 \\
\hline $\mathrm{O}$ & -1.761962 & 1.619904 & -1.812715 \\
\hline $\mathrm{C}$ & -0.446131 & 1.618264 & -2.345715 \\
\hline $\mathrm{C}$ & -1.276611 & 3.432259 & -0.231084 \\
\hline $\mathrm{C}$ & -3.294899 & 1.908978 & -0.059553 \\
\hline $\mathrm{H}$ & 5.253789 & -1.290226 & -2.229601 \\
\hline $\mathrm{H}$ & 2.975943 & 1.951084 & -0.020536 \\
\hline $\mathrm{H}$ & -0.757504 & -1.673995 & -1.313144 \\
\hline $\mathrm{H}$ & -0.238218 & -2.835714 & -0.102853 \\
\hline $\mathrm{H}$ & -2.016710 & -1.600847 & 1.495001 \\
\hline $\mathrm{H}$ & -2.517991 & -2.353365 & -2.186734 \\
\hline $\mathrm{H}$ & -3.831738 & -3.436101 & -1.708104 \\
\hline $\mathrm{H}$ & -4.174338 & -1.743515 & -2.048196 \\
\hline $\mathrm{H}$ & -4.291512 & -1.845743 & 1.740458 \\
\hline $\mathrm{H}$ & -5.224601 & -1.364352 & 0.308559 \\
\hline $\mathrm{H}$ & -4.983791 & -3.071763 & 0.660027 \\
\hline $\mathrm{H}$ & 2.869814 & -0.766173 & 2.549129 \\
\hline $\mathrm{H}$ & 2.725360 & 0.724738 & 3.513255 \\
\hline $\mathrm{H}$ & 1.848906 & -0.742287 & 3.984881 \\
\hline
\end{tabular}




$\begin{array}{rrrr}\mathrm{H} & -0.378033 & 0.177047 & 4.009923 \\ \mathrm{H} & 0.338318 & 1.780324 & 3.757347 \\ \mathrm{H} & -0.973720 & 1.211660 & 2.694571 \\ \mathrm{H} & 1.055832 & 2.231824 & 1.310932 \\ \mathrm{H} & 3.837364 & -2.288787 & -1.792835 \\ \mathrm{H} & 3.685157 & -0.985285 & -3.008048 \\ \mathrm{H} & 2.159676 & 0.633053 & -2.795604 \\ \mathrm{H} & -0.512012 & 1.093086 & -3.303750 \\ \mathrm{H} & -0.117212 & 2.647113 & -2.562832 \\ \mathrm{H} & -0.204917 & 3.501596 & -0.438624 \\ \mathrm{H} & -1.798935 & 4.142956 & -0.879826 \\ \mathrm{H} & -1.434074 & 3.727592 & 0.810226 \\ \mathrm{H} & -3.891914 & 2.606741 & -0.653860 \\ \mathrm{H} & -3.638558 & 0.888651 & -0.241248 \\ \mathrm{H} & -3.419592 & 2.142986 & 1.001356 \\ \mathrm{H} & 3.398546 & 0.733976 & 1.177401\end{array}$

Sum of Energies $=-1194.346627$

\section{TS}

$\begin{array}{lrrr}\mathrm{C} & -4.026017 & 2.065001 & -1.745656 \\ \mathrm{O} & -3.751352 & 0.705531 & -1.382585 \\ \mathrm{C} & -2.449786 & 0.420380 & -1.031586 \\ \mathrm{C} & -1.573872 & -0.060708 & -2.028130 \\ \mathrm{C} & -0.327453 & -0.548492 & -1.684131 \\ \mathrm{C} & 0.058333 & -0.505274 & -0.325577 \\ \mathrm{C} & -1.475844 & -1.643191 & 0.779761 \\ \mathrm{C} & -2.700102 & -1.319372 & 0.174129 \\ \mathrm{C} & -1.856798 & 1.088541 & 0.151316 \\ \mathrm{C} & -0.411772 & 0.643754 & 0.558565 \\ \mathrm{O} & -2.451240 & 1.915619 & 0.825983 \\ \mathrm{C} & 0.475785 & 1.914458 & 0.460008 \\ \mathrm{C} & 1.880368 & 1.764778 & 0.975137 \\ \mathrm{C} & 2.999292 & 2.305207 & 0.467608 \\ \mathrm{C} & 3.069564 & 3.145856 & -0.784450 \\ \mathrm{C} & 4.326322 & 2.115388 & 1.165772 \\ \mathrm{O} & -0.403424 & 0.237287 & 1.912144 \\ \mathrm{C} & -1.071543 & -1.049719 & 2.133834 \\ \mathrm{C} & -2.256310 & -0.753087 & 3.068462 \\ \mathrm{C} & -0.061585 & -1.954758 & 2.848545 \\ \mathrm{O} & 1.271747 & -1.003043 & 0.058310 \\ \mathrm{C} & 2.035087 & -1.801062 & -0.885344 \\ \mathrm{O} & 1.916103 & -1.278843 & -2.188738 \\ \mathrm{C} & 0.578087 & -1.231593 & -2.677400 \\ \mathrm{C} & 1.586884 & -3.264418 & -0.793944 \\ \mathrm{C} & 3.489191 & -1.614125 & -0.480802\end{array}$




$\begin{array}{lrrr}\mathrm{H} & -5.077905 & 2.087040 & -2.039878 \\ \mathrm{H} & -3.065633 & -1.939728 & -0.639187 \\ \mathrm{H} & 0.451932 & 2.267465 & -0.576489 \\ \mathrm{H} & -0.060968 & 2.661831 & 1.060738 \\ \mathrm{H} & 1.959662 & 1.189261 & 1.894268 \\ \mathrm{H} & 2.111572 & 3.235727 & -1.302223 \\ \mathrm{H} & 3.416191 & 4.162595 & -0.550497 \\ \mathrm{H} & 3.796927 & 2.727188 & -1.494025 \\ \mathrm{H} & 4.228160 & 1.500108 & 2.065552 \\ \mathrm{H} & 5.061222 & 1.635492 & 0.503029 \\ \mathrm{H} & 4.761885 & 3.081076 & 1.460373 \\ \mathrm{H} & -2.927985 & -0.005127 & 2.641672 \\ \mathrm{H} & -2.824416 & -1.664633 & 3.285421 \\ \mathrm{H} & -1.874664 & -0.347807 & 4.011288 \\ \mathrm{H} & 0.289592 & -1.467046 & 3.763675 \\ \mathrm{H} & -0.526123 & -2.909036 & 3.124105 \\ \mathrm{H} & 0.801719 & -2.148970 & 2.209008 \\ \mathrm{H} & -1.007640 & -2.585209 & 0.512441 \\ \mathrm{H} & -3.858673 & 2.739512 & -0.903222 \\ \mathrm{H} & -3.407261 & 2.373034 & -2.600152 \\ \mathrm{H} & -1.967557 & -0.191348 & -3.034247 \\ \mathrm{H} & 0.626632 & -0.660084 & -3.609364 \\ \mathrm{H} & 0.224304 & -2.242985 & -2.934409 \\ \mathrm{H} & 0.524997 & -3.385519 & -1.022287 \\ \mathrm{H} & 2.163991 & -3.868449 & -1.501257 \\ \mathrm{H} & 1.759725 & -3.644837 & 0.216538 \\ \mathrm{H} & 4.136386 & -2.206358 & -1.134094 \\ \mathrm{H} & 3.753173 & -0.558218 & -0.569235 \\ \mathrm{H} & 3.635663 & -1.932093 & 0.554949 \\ \mathrm{H} & -3.486568 & -0.820673 & 0.729878\end{array}$

Sum of Energies $=-1194.289230$

16

$\begin{array}{lrrr}\mathrm{C} & -2.041151 & -2.729587 & -2.575551 \\ \mathrm{O} & -2.026113 & -1.590804 & -1.725234 \\ \mathrm{C} & -0.865694 & -1.268171 & -1.112173 \\ \mathrm{C} & 0.315994 & -1.912025 & -1.256453 \\ \mathrm{C} & 1.498378 & -1.470054 & -0.544257 \\ \mathrm{C} & 1.482386 & -0.399990 & 0.295740 \\ \mathrm{C} & 0.227444 & 0.300550 & 0.615502 \\ \mathrm{C} & -0.984760 & 0.025086 & -0.314190 \\ \mathrm{O} & -2.239301 & 0.188676 & 0.307365 \\ \mathrm{C} & -2.689149 & -0.542843 & 1.479668 \\ \mathrm{C} & -2.791071 & 0.483709 & 2.625346 \\ \mathrm{C} & -1.788547 & -1.718891 & 1.896088\end{array}$




\begin{tabular}{|c|c|c|c|}
\hline C & -4.080665 & -1.073112 & 1.178837 \\
\hline $\mathrm{C}$ & -4.748296 & -0.955760 & 0.033319 \\
\hline $\mathrm{O}$ & 0.153109 & 1.121053 & 1.516832 \\
\hline C & -0.940531 & 1.183630 & -1.397725 \\
\hline C & -1.144847 & 2.557216 & -0.832356 \\
\hline C & -0.290733 & 3.592524 & -0.852684 \\
\hline $\mathrm{C}$ & 1.096943 & 3.568125 & -1.444158 \\
\hline C & -0.680710 & 4.915729 & -0.238508 \\
\hline C & 2.783406 & -2.238214 & -0.700779 \\
\hline $\mathrm{O}$ & 3.726041 & -1.902074 & 0.308642 \\
\hline $\mathrm{C}$ & 3.837467 & -0.514424 & 0.577522 \\
\hline $\mathrm{O}$ & 2.570718 & 0.022315 & 1.004882 \\
\hline $\mathrm{C}$ & 4.765758 & -0.389329 & 1.774556 \\
\hline C & 4.328396 & 0.275432 & -0.641219 \\
\hline $\mathrm{H}$ & -3.065206 & -2.817362 & -2.940525 \\
\hline $\mathrm{H}$ & -3.214486 & 0.021141 & 3.524934 \\
\hline $\mathrm{H}$ & -1.803349 & 0.888892 & 2.853214 \\
\hline $\mathrm{H}$ & -3.445008 & 1.306679 & 2.321276 \\
\hline $\mathrm{H}$ & -1.677156 & -2.451471 & 1.092324 \\
\hline $\mathrm{H}$ & -0.800476 & -1.383023 & 2.221377 \\
\hline $\mathrm{H}$ & -2.252715 & -2.230433 & 2.746378 \\
\hline $\mathrm{H}$ & -4.550456 & -1.569470 & 2.029331 \\
\hline $\mathrm{H}$ & -5.755102 & -1.352214 & -0.071338 \\
\hline $\mathrm{H}$ & -4.307703 & -0.464138 & -0.826228 \\
\hline $\mathrm{H}$ & -0.001878 & 1.091743 & -1.951830 \\
\hline $\mathrm{H}$ & -1.756184 & 0.941566 & -2.090326 \\
\hline $\mathrm{H}$ & -2.108931 & 2.700601 & -0.350807 \\
\hline $\mathrm{H}$ & 1.213151 & 4.358703 & -2.198694 \\
\hline $\mathrm{H}$ & 1.845279 & 3.767408 & -0.664790 \\
\hline $\mathrm{H}$ & 1.355423 & 2.616689 & -1.915235 \\
\hline $\mathrm{H}$ & -0.638586 & 5.727336 & -0.979090 \\
\hline $\mathrm{H}$ & -1.690883 & 4.887673 & 0.180913 \\
\hline $\mathrm{H}$ & 0.013462 & 5.191681 & 0.567577 \\
\hline $\mathrm{H}$ & -1.354166 & -2.600179 & -3.421801 \\
\hline $\mathrm{H}$ & -1.767687 & -3.636852 & -2.022471 \\
\hline $\mathrm{H}$ & 2.601359 & -3.313035 & -0.584583 \\
\hline $\mathrm{H}$ & 3.209378 & -2.091862 & -1.708556 \\
\hline $\mathrm{H}$ & 4.821215 & 0.653684 & 2.097141 \\
\hline $\mathrm{H}$ & 4.378871 & -0.996365 & 2.596745 \\
\hline $\mathrm{H}$ & 5.767648 & -0.740346 & 1.511475 \\
\hline $\mathrm{H}$ & 4.486065 & 1.321655 & -0.364807 \\
\hline $\mathrm{H}$ & 5.273425 & -0.143731 & -1.001144 \\
\hline $\mathrm{H}$ & 3.599851 & 0.250277 & -1.456595 \\
\hline $\mathrm{H}$ & 0.409101 & -2.786173 & -1.892920 \\
\hline
\end{tabular}

Sum of Energies $=-1194.323772$ 


\section{TS}

\begin{tabular}{|c|c|c|c|}
\hline $\mathrm{C}$ & -2.049813 & -3.561629 & -1.488779 \\
\hline 0 & -2.070921 & -2.217350 & -1.041398 \\
\hline $\mathrm{C}$ & -0.894753 & -1.670361 & -0.611136 \\
\hline $\mathrm{C}$ & 0.321924 & -2.315518 & -0.604632 \\
\hline $\mathrm{C}$ & 1.485431 & -1.658609 & -0.117905 \\
\hline $\mathrm{C}$ & 1.430332 & -0.349761 & 0.301025 \\
\hline $\mathrm{C}$ & 0.172792 & 0.378710 & 0.362760 \\
\hline $\mathrm{C}$ & -0.994934 & -0.305934 & -0.167293 \\
\hline 0 & -2.246107 & 0.231996 & -0.013604 \\
\hline $\mathrm{C}$ & -2.938070 & 0.220913 & 1.275676 \\
\hline $\mathrm{C}$ & -2.894301 & 1.652365 & 1.838405 \\
\hline $\mathrm{C}$ & -2.326438 & -0.776001 & 2.271744 \\
\hline $\mathrm{C}$ & -4.379339 & -0.162457 & 1.000730 \\
\hline $\mathrm{C}$ & -4.886613 & -0.565052 & -0.162222 \\
\hline 0 & 0.112720 & 1.579607 & 0.762787 \\
\hline $\mathrm{C}$ & 0.073922 & 2.920356 & -1.107463 \\
\hline $\mathrm{C}$ & 1.553387 & 2.804641 & -1.341497 \\
\hline $\mathrm{C}$ & -0.368147 & 4.181360 & -0.423005 \\
\hline $\mathrm{C}$ & -0.869749 & 2.109777 & -1.738118 \\
\hline $\mathrm{C}$ & -0.625946 & 0.824919 & -2.200048 \\
\hline $\mathrm{C}$ & 2.799018 & -2.392994 & -0.023176 \\
\hline 0 & 3.714183 & -1.707528 & 0.825973 \\
\hline $\mathrm{C}$ & 3.799530 & -0.314797 & 0.589448 \\
\hline $\mathrm{C}$ & 4.305529 & 0.000044 & -0.824445 \\
\hline $\mathrm{C}$ & 4.709991 & 0.241605 & 1.673394 \\
\hline 0 & 2.522969 & 0.320393 & 0.787553 \\
\hline $\mathrm{H}$ & -3.077835 & -3.801220 & -1.766614 \\
\hline $\mathrm{H}$ & -3.461079 & 1.714465 & 2.775259 \\
\hline $\mathrm{H}$ & -1.859652 & 1.952796 & 2.012538 \\
\hline $\mathrm{H}$ & -3.344858 & 2.344921 & 1.120029 \\
\hline $\mathrm{H}$ & -2.322839 & -1.789348 & 1.860410 \\
\hline $\mathrm{H}$ & -1.305724 & -0.493391 & 2.545786 \\
\hline $\mathrm{H}$ & -2.929498 & -0.784544 & 3.186228 \\
\hline $\mathrm{H}$ & -5.028558 & -0.069598 & 1.872625 \\
\hline $\mathrm{H}$ & -5.942035 & -0.807040 & -0.258717 \\
\hline $\mathrm{H}$ & -4.261041 & -0.670281 & -1.041004 \\
\hline $\mathrm{H}$ & 1.858232 & 3.551968 & -2.089792 \\
\hline $\mathrm{H}$ & 2.104112 & 3.003637 & -0.419328 \\
\hline $\mathrm{H}$ & 1.853102 & 1.823040 & -1.710885 \\
\hline $\mathrm{H}$ & -1.454314 & 4.225718 & -0.308520 \\
\hline $\mathrm{H}$ & 0.088220 & 4.240042 & 0.570723 \\
\hline $\mathrm{H}$ & -0.038618 & 5.065865 & -0.987749 \\
\hline $\mathrm{H}$ & -1.912200 & 2.398490 & -1.632226 \\
\hline $\mathrm{H}$ & -1.433445 & 0.251312 & -2.642104 \\
\hline $\mathrm{H}$ & 0.374925 & 0.456893 & -2.396043 \\
\hline $\mathrm{H}$ & -1.396371 & -3.686449 & -2.363256 \\
\hline
\end{tabular}




$\begin{array}{rrrr}\mathrm{H} & -1.718492 & -4.245111 & -0.695441 \\ \mathrm{H} & 0.410276 & -3.342979 & -0.941324 \\ \mathrm{H} & 2.659050 & -3.376771 & 0.438136 \\ \mathrm{H} & 3.242715 & -2.563371 & -1.018666 \\ \mathrm{H} & 4.467051 & 1.076412 & -0.931302 \\ \mathrm{H} & 5.252079 & -0.519390 & -1.005711 \\ \mathrm{H} & 3.584624 & -0.309669 & -1.586663 \\ \mathrm{H} & 4.747071 & 1.332428 & 1.608929 \\ \mathrm{H} & 5.720619 & -0.161264 & 1.560269 \\ \mathrm{H} & 4.319219 & -0.043969 & 2.653161\end{array}$

Sum of Energies $=-1194.290386$

17

\begin{tabular}{|c|c|c|c|}
\hline $\mathrm{O}$ & 1.700403 & -2.064096 & 1.646767 \\
\hline $\mathrm{C}$ & 0.620840 & -1.429157 & 0.973824 \\
\hline $\mathrm{C}$ & -0.476549 & -0.901420 & 1.864132 \\
\hline $\mathrm{C}$ & -1.526757 & -0.335480 & 1.257005 \\
\hline C & -1.473079 & -0.420893 & -0.254806 \\
\hline $\mathrm{C}$ & -1.285382 & -1.929892 & -0.594833 \\
\hline C & 0.106910 & -2.415249 & -0.143000 \\
\hline C & 1.114308 & -0.299698 & -0.002414 \\
\hline $\mathrm{C}$ & -0.165130 & 0.326194 & -0.644153 \\
\hline $\mathrm{C}$ & 2.619347 & 1.737847 & -0.362410 \\
\hline $\mathrm{C}$ & 2.813046 & 3.050414 & -0.169371 \\
\hline C & 2.460337 & 3.806930 & 1.088307 \\
\hline $\mathrm{C}$ & 3.428276 & 3.901768 & -1.254819 \\
\hline $\mathrm{C}$ & 1.229489 & -2.315060 & -1.234666 \\
\hline C & 2.336315 & -3.355712 & -1.015721 \\
\hline C & 0.754857 & -2.384587 & -2.692288 \\
\hline C & 2.062886 & 0.734866 & 0.615464 \\
\hline $\mathrm{O}$ & -0.143400 & 1.269045 & -1.401304 \\
\hline $\mathrm{O}$ & 1.812787 & -0.995482 & -1.032544 \\
\hline C & 2.013357 & -1.706363 & 2.982857 \\
\hline $\mathrm{C}$ & -2.701500 & 0.345115 & 1.910637 \\
\hline $\mathrm{O}$ & -3.813165 & 0.427157 & 1.029412 \\
\hline C & -3.504373 & 0.935151 & -0.265099 \\
\hline $\mathrm{O}$ & -2.609083 & 0.044694 & -0.944549 \\
\hline $\mathrm{C}$ & -2.938543 & 2.359159 & -0.214408 \\
\hline C & -4.805784 & 0.865764 & -1.051883 \\
\hline $\mathrm{H}$ & -2.074494 & -2.474614 & -0.068486 \\
\hline $\mathrm{H}$ & 0.052132 & -3.430546 & 0.256038 \\
\hline $\mathrm{H}$ & 2.920868 & 1.310133 & -1.315972 \\
\hline $\mathrm{H}$ & 1.989323 & 3.185640 & 1.854517 \\
\hline $\mathrm{H}$ & 3.357097 & 4.259684 & 1.535126 \\
\hline $\mathrm{H}$ & 1.775882 & 4.636657 & 0.862135 \\
\hline
\end{tabular}




$\begin{array}{rrrr}\mathrm{H} & 3.669554 & 3.313368 & -2.145326 \\ \mathrm{H} & 2.746076 & 4.709086 & -1.557284 \\ \mathrm{H} & 4.350992 & 4.388268 & -0.906412 \\ \mathrm{H} & 2.684691 & -3.345299 & 0.017169 \\ \mathrm{H} & 1.969764 & -4.360620 & -1.258544 \\ \mathrm{H} & 3.180682 & -3.133134 & -1.676759 \\ \mathrm{H} & 1.627204 & -2.347733 & -3.352927 \\ \mathrm{H} & 0.216720 & -3.318408 & -2.893695 \\ \mathrm{H} & 0.111702 & -1.541278 & -2.956860 \\ \mathrm{H} & -1.454029 & -2.069124 & -1.663242 \\ \mathrm{H} & 1.551772 & 1.227932 & 1.449695 \\ \mathrm{H} & 2.891388 & 0.148350 & 1.036536 \\ \mathrm{H} & -0.386897 & -0.947353 & 2.944346 \\ \mathrm{H} & 2.995682 & -2.142101 & 3.183751 \\ \mathrm{H} & 2.075193 & -0.621555 & 3.133740 \\ \mathrm{H} & 1.296785 & -2.126931 & 3.702533 \\ \mathrm{H} & -3.047414 & -0.221924 & 2.780992 \\ \mathrm{H} & -2.416096 & 1.347296 & 2.268009 \\ \mathrm{H} & -2.766214 & 2.716668 & -1.232027 \\ \mathrm{H} & -3.648982 & 3.023171 & 0.289217 \\ \mathrm{H} & -1.980028 & 2.411568 & 0.306824 \\ \mathrm{H} & -5.183950 & -0.159509 & -1.033915 \\ \mathrm{H} & -5.551955 & 1.529764 & -0.606475 \\ \mathrm{H} & -4.631014 & 1.163812 & -2.089093\end{array}$

Sum of Energies $=-1194.342602$

\section{TS}

$\begin{array}{lrrr}\text { O } & 1.719331 & -1.907257 & 1.746401 \\ \mathrm{C} & 0.674713 & -1.269957 & 1.121275 \\ \mathrm{C} & -0.562915 & -1.079734 & 1.768527 \\ \mathrm{C} & -1.597021 & -0.455379 & 1.091960 \\ \mathrm{C} & -1.372491 & -0.024210 & -0.235200 \\ \mathrm{C} & -0.960798 & -1.932053 & -1.141646 \\ \mathrm{C} & 0.264391 & -2.415268 & -0.650357 \\ \mathrm{C} & 1.184225 & -0.209913 & 0.149403 \\ \mathrm{C} & -0.006112 & 0.416488 & -0.635682 \\ \mathrm{C} & 2.589875 & 1.940268 & 0.045558 \\ \mathrm{C} & 2.587816 & 3.269746 & 0.223972 \\ \mathrm{C} & 1.900498 & 3.997658 & 1.353628 \\ \mathrm{C} & 3.313430 & 4.172492 & -0.745690 \\ \mathrm{C} & 1.590797 & -2.051852 & -1.333674 \\ \mathrm{C} & 2.652459 & -3.126387 & -1.071874 \\ \mathrm{C} & 1.471813 & -1.795172 & -2.845142 \\ \mathrm{C} & 1.958725 & 0.893834 & 0.921669 \\ \mathrm{O} & 0.184325 & 1.180792 & -1.563240 \\ & & 44 & \end{array}$




$\begin{array}{rrrr}\mathrm{O} & 2.099114 & -0.808690 & -0.747744 \\ \mathrm{C} & 1.425739 & -2.797246 & 2.811861 \\ \mathrm{C} & -3.006017 & -0.403909 & 1.632268 \\ \mathrm{O} & -3.953719 & -0.224908 & 0.585101 \\ \mathrm{C} & -3.624733 & 0.803304 & -0.332386 \\ \mathrm{O} & -2.377600 & 0.513120 & -0.996928 \\ \mathrm{C} & -3.512137 & 2.177500 & 0.336818 \\ \mathrm{C} & -4.691910 & 0.761040 & -1.414139 \\ \mathrm{H} & -1.884978 & -2.395185 & -0.807702 \\ \mathrm{H} & 0.257192 & -3.348531 & -0.093090 \\ \mathrm{H} & 3.123167 & 1.539597 & -0.813191 \\ \mathrm{H} & 1.354176 & 3.336707 & 2.031380 \\ \mathrm{H} & 2.629238 & 4.559494 & 1.955402 \\ \mathrm{H} & 1.188995 & 4.737468 & 0.960638 \\ \mathrm{H} & 3.795840 & 3.603902 & -1.546436 \\ \mathrm{H} & 2.620927 & 4.889520 & -1.209375 \\ \mathrm{H} & 4.084505 & 4.769090 & -0.236629 \\ \mathrm{H} & 2.771149 & -3.297813 & 0.000021 \\ \mathrm{H} & 2.374923 & -4.069384 & -1.557948 \\ \mathrm{H} & 3.616093 & -2.801301 & -1.477300 \\ \mathrm{H} & 2.465575 & -1.573279 & -3.247903 \\ \mathrm{H} & 1.074260 & -2.674094 & -3.365055 \\ \mathrm{H} & 0.835947 & -0.933293 & -3.058031 \\ \mathrm{H} & -1.029520 & -1.470443 & -2.120430 \\ \mathrm{H} & 1.279878 & 1.331666 & 1.660790 \\ \mathrm{H} & 2.737314 & 0.355645 & 1.478245 \\ \mathrm{H} & -0.766071 & -1.555388 & 2.722822 \\ \mathrm{H} & 2.356970 & -3.323893 & 3.029738 \\ \mathrm{H} & 1.100067 & -2.259579 & 3.712097 \\ \mathrm{H} & -3.652243 & -3.523403 & 2.528025 \\ \mathrm{H} & -3.278839 & -1.348802 & 2.113846 \\ \mathrm{H} & -3.108779 & 0.388801 & 2.390582 \\ \mathrm{H} & -4.430356 & 2.404880 & 0.888111 \\ \mathrm{H} & -2.665496 & 2.221097 & 1.028162 \\ \mathrm{H} & -746918 & -0.248596 & -1.829142 \\ \mathrm{H} & \mathrm{H} & 1.026590 & -0.992597 \\ \mathrm{H} & -36508 & 1.462594 & -2.214097\end{array}$

Sum of Energies $=-1194.288609$

\section{Pink Pathway (Claisen Intermediate)}

$\begin{array}{lrrr}\mathrm{C} & -1.750084 & -0.445092 & 3.448602 \\ \mathrm{O} & -2.367607 & -0.743741 & 2.204930 \\ \mathrm{C} & -1.577218 & -0.480206 & 1.051431 \\ \mathrm{C} & -1.391951 & 0.997068 & 0.844924\end{array}$




\begin{tabular}{|c|c|c|c|}
\hline C & -0.225834 & 1.548516 & 0.472032 \\
\hline $\mathrm{C}$ & 0.970389 & 0.713589 & 0.314043 \\
\hline $\mathrm{C}$ & 0.963596 & -0.634752 & 0.574361 \\
\hline C & -0.249247 & -1.284192 & 1.072724 \\
\hline $\mathrm{O}$ & -0.265352 & -2.452942 & 1.449760 \\
\hline $\mathrm{O}$ & 2.139148 & -1.324701 & 0.607190 \\
\hline $\mathrm{C}$ & 2.506752 & -2.284222 & -0.453438 \\
\hline $\mathrm{C}$ & 2.970373 & -3.530689 & 0.311879 \\
\hline C & 3.663997 & -1.637338 & -1.218392 \\
\hline $\mathrm{C}$ & 1.320683 & -2.644764 & -1.322139 \\
\hline $\mathrm{C}$ & 1.210514 & -2.441435 & -2.635650 \\
\hline C & -2.394988 & -1.078452 & -0.143256 \\
\hline $\mathrm{C}$ & -3.776986 & -0.504912 & -0.282380 \\
\hline C & -4.302958 & 0.144572 & -1.333708 \\
\hline C & -3.573090 & 0.457403 & -2.617379 \\
\hline C & -5.738358 & 0.615790 & -1.302661 \\
\hline C & -0.075064 & 3.031789 & 0.211827 \\
\hline O & 1.060846 & 3.303773 & -0.604082 \\
\hline C & 2.254605 & 2.713416 & -0.149579 \\
\hline $\mathrm{O}$ & 2.127368 & 1.266363 & -0.118863 \\
\hline $\mathrm{C}$ & 2.675274 & 3.186154 & 1.246193 \\
\hline $\mathrm{C}$ & 3.305585 & 3.002930 & -1.209326 \\
\hline $\mathrm{H}$ & -2.509894 & -0.618099 & 4.214875 \\
\hline $\mathrm{H}$ & 0.519358 & -3.152692 & -0.790004 \\
\hline $\mathrm{H}$ & 0.331284 & -2.775375 & -3.180870 \\
\hline $\mathrm{H}$ & 1.980534 & -1.948696 & -3.222603 \\
\hline $\mathrm{H}$ & -4.413191 & -0.663708 & 0.586599 \\
\hline $\mathrm{H}$ & -2.287939 & 1.604876 & 0.949607 \\
\hline $\mathrm{H}$ & -0.892488 & -1.101275 & 3.640383 \\
\hline $\mathrm{H}$ & -1.422981 & 0.604209 & 3.502015 \\
\hline $\mathrm{H}$ & 3.327092 & -4.301840 & -0.379675 \\
\hline $\mathrm{H}$ & 3.785115 & -3.265632 & 0.994177 \\
\hline $\mathrm{H}$ & 2.141190 & -3.929289 & 0.902672 \\
\hline $\mathrm{H}$ & 4.081511 & -2.326344 & -1.960717 \\
\hline $\mathrm{H}$ & 3.339477 & -0.722700 & -1.722114 \\
\hline $\mathrm{H}$ & 4.454303 & -1.369670 & -0.510917 \\
\hline $\mathrm{H}$ & -1.800940 & -0.967433 & -1.055116 \\
\hline $\mathrm{H}$ & -2.455854 & -2.151790 & 0.081472 \\
\hline $\mathrm{H}$ & -3.595126 & 1.537304 & -2.820325 \\
\hline $\mathrm{H}$ & -2.527600 & 0.141403 & -2.612351 \\
\hline $\mathrm{H}$ & -4.067069 & -0.025928 & -3.471958 \\
\hline $\mathrm{H}$ & -5.802797 & 1.703702 & -1.447997 \\
\hline $\mathrm{H}$ & -6.321569 & 0.160953 & -2.115968 \\
\hline $\mathrm{H}$ & -6.228520 & 0.368961 & -0.355848 \\
\hline $\mathrm{H}$ & -0.934329 & 3.418244 & -0.341990 \\
\hline $\mathrm{H}$ & -0.011855 & 3.591739 & 1.156939 \\
\hline $\mathrm{H}$ & 3.647543 & 2.755459 & 1.499678 \\
\hline $\mathrm{H}$ & 2.753958 & 4.277740 & 1.262846 \\
\hline
\end{tabular}




$\begin{array}{lllr}\mathrm{H} & 1.964286 & 2.872183 & 2.015775 \\ \mathrm{H} & 4.237046 & 2.484265 & -0.968519 \\ \mathrm{H} & 2.943559 & 2.659564 & -2.181677 \\ \mathrm{H} & 3.494733 & 4.078817 & -1.260518\end{array}$

Sum of Energies $=-1194.324623$

\section{Pink Pathway (Claisen TS)}

\begin{tabular}{|c|c|c|c|}
\hline $\mathrm{C}$ & 2.162035 & -2.455705 & 2.439602 \\
\hline $\mathrm{O}$ & 1.690104 & -2.804334 & 1.134427 \\
\hline $\mathrm{C}$ & 0.661905 & -2.007111 & 0.669433 \\
\hline C & -0.604241 & -2.570164 & 0.554846 \\
\hline C & -1.685364 & -1.821074 & 0.088504 \\
\hline C & -1.501681 & -0.465767 & -0.203420 \\
\hline C & -0.226233 & 0.129236 & -0.083532 \\
\hline $\mathrm{C}$ & 0.879320 & -0.667697 & 0.279349 \\
\hline $\mathrm{O}$ & 2.129217 & -0.106426 & 0.389529 \\
\hline C & 3.063384 & -0.092136 & -0.754220 \\
\hline C & 4.143991 & 0.889045 & -0.281953 \\
\hline C & 2.386000 & 0.415069 & -2.032416 \\
\hline $\mathrm{C}$ & 3.646498 & -1.485940 & -0.954091 \\
\hline $\mathrm{C}$ & 4.517376 & -1.824032 & -1.906051 \\
\hline $\mathrm{O}$ & -0.049406 & 1.438007 & -0.448300 \\
\hline C & -0.102232 & 2.531690 & 0.540094 \\
\hline C & 0.381603 & 2.095342 & 1.922077 \\
\hline C & -1.546813 & 3.055521 & 0.608160 \\
\hline C & 0.771372 & 3.590666 & -0.101947 \\
\hline C & 1.702529 & 4.338452 & 0.489628 \\
\hline C & -3.050359 & -2.428520 & -0.123072 \\
\hline $\mathrm{O}$ & -3.874034 & -1.581296 & -0.920064 \\
\hline C & -3.860042 & -0.223854 & -0.532406 \\
\hline C & -4.366291 & -0.019573 & 0.901012 \\
\hline $\mathrm{C}$ & -4.694925 & 0.520097 & -1.562815 \\
\hline $\mathrm{O}$ & -2.527862 & 0.324932 & -0.644890 \\
\hline $\mathrm{H}$ & 2.936704 & -3.185208 & 2.688158 \\
\hline $\mathrm{H}$ & 4.936007 & 0.966186 & -1.032959 \\
\hline $\mathrm{H}$ & 3.707496 & 1.879410 & -0.118164 \\
\hline $\mathrm{H}$ & 4.592545 & 0.539826 & 0.653431 \\
\hline $\mathrm{H}$ & 1.580130 & -0.254837 & -2.345976 \\
\hline $\mathrm{H}$ & 1.973451 & 1.413646 & -1.883471 \\
\hline $\mathrm{H}$ & 3.124016 & 0.446096 & -2.840254 \\
\hline $\mathrm{H}$ & 3.309806 & -2.234143 & -0.242644 \\
\hline $\mathrm{H}$ & 4.903160 & -2.837571 & -1.976922 \\
\hline $\mathrm{H}$ & 4.887123 & -1.119857 & -2.648841 \\
\hline $\mathrm{H}$ & 0.341346 & 2.944710 & 2.611692 \\
\hline $\mathrm{H}$ & -0.268705 & 1.313482 & 2.326014 \\
\hline
\end{tabular}




$\begin{array}{lrrr}\mathrm{H} & 1.403538 & 1.714585 & 1.879711 \\ \mathrm{H} & -1.909606 & 3.304253 & -0.393259 \\ \mathrm{H} & -2.212026 & 2.298746 & 1.031556 \\ \mathrm{H} & -1.589825 & 3.955159 & 1.232348 \\ \mathrm{H} & 0.553889 & 3.739672 & -1.159555 \\ \mathrm{H} & 2.248064 & 5.096050 & -0.066621 \\ \mathrm{H} & 1.959373 & 4.236367 & 1.540333 \\ \mathrm{H} & 2.586244 & -1.446622 & 2.450774 \\ \mathrm{H} & 1.349683 & -2.522978 & 3.175518 \\ \mathrm{H} & -0.727104 & -3.616228 & 0.823202 \\ \mathrm{H} & -2.974933 & -3.366222 & -0.682735 \\ \mathrm{H} & -3.544459 & -2.659756 & 0.834645 \\ \mathrm{H} & -4.457618 & 1.049167 & 1.114577 \\ \mathrm{H} & -5.348964 & -0.487379 & 1.018866 \\ \mathrm{H} & -3.681183 & -0.452625 & 1.635594 \\ \mathrm{H} & -4.652327 & 1.596540 & -1.376415 \\ \mathrm{H} & -5.735447 & 0.186746 & -1.513550 \\ \mathrm{H} & -4.301514 & 0.314451 & -2.561360\end{array}$

Sum of Energies $=-1194.328294$

\section{Pink Pathway (Diels-Alder Product)}

\begin{tabular}{|c|c|c|c|}
\hline $\mathrm{C}$ & 0.565430 & -3.914266 & -0.640475 \\
\hline $\mathrm{O}$ & -0.237373 & -2.986696 & 0.067887 \\
\hline $\mathrm{C}$ & 0.222270 & -1.633554 & 0.188622 \\
\hline C & 0.110557 & -0.821182 & -1.148592 \\
\hline C & 0.311384 & 0.645429 & -0.760276 \\
\hline $\mathrm{C}$ & -0.572218 & 1.143966 & 0.135004 \\
\hline C & -1.648924 & 0.133374 & 0.448777 \\
\hline C & -0.860116 & -1.013064 & 1.119211 \\
\hline C & -1.331186 & -1.107783 & -1.733617 \\
\hline C & -2.276348 & -0.236507 & -0.907212 \\
\hline 0 & -2.930543 & 0.354414 & 1.029789 \\
\hline C & -3.571367 & -0.446626 & -0.059920 \\
\hline C & -3.880026 & -1.865582 & 0.405534 \\
\hline C & -4.815173 & 0.275051 & -0.553047 \\
\hline C & 1.601060 & -1.538477 & 0.904336 \\
\hline C & 2.836731 & -1.681400 & 0.050055 \\
\hline C & 3.980779 & -0.981763 & 0.149463 \\
\hline C & 5.150034 & -1.292573 & -0.756340 \\
\hline $\mathrm{C}$ & 4.240435 & 0.128525 & 1.138853 \\
\hline $\mathrm{O}$ & -1.042304 & -1.377944 & 2.256897 \\
\hline C & 1.461380 & 1.509704 & -1.182383 \\
\hline $\mathrm{O}$ & 1.597499 & 2.610286 & -0.269597 \\
\hline $\mathrm{C}$ & 0.402236 & 3.289060 & 0.045237 \\
\hline $\mathrm{O}$ & -0.566439 & 2.385094 & 0.665073 \\
\hline
\end{tabular}




$\begin{array}{lrrr}\mathrm{C} & -0.264441 & 3.915979 & -1.181985 \\ \mathrm{C} & 0.754150 & 4.305415 & 1.118385 \\ \mathrm{H} & -0.036375 & -4.822459 & -0.734132 \\ \mathrm{H} & -2.422435 & 0.710181 & -1.435982 \\ \mathrm{H} & -4.558165 & -1.831246 & 1.264785 \\ \mathrm{H} & -4.373579 & -2.422963 & -0.400036 \\ \mathrm{H} & -2.985532 & -2.417364 & 0.701073 \\ \mathrm{H} & -5.231928 & -0.231854 & -1.431728 \\ \mathrm{H} & -5.582379 & 0.287687 & 0.229286 \\ \mathrm{H} & -4.582844 & 1.310403 & -0.820592 \\ \mathrm{H} & 1.635007 & -0.589512 & 1.443944 \\ \mathrm{H} & 1.571894 & -2.315947 & 1.680367 \\ \mathrm{H} & 2.814564 & -2.464530 & -0.705736 \\ \mathrm{H} & 5.449800 & -0.407304 & -1.334734 \\ \mathrm{H} & 6.031824 & -1.592969 & -0.173118 \\ \mathrm{H} & 4.920944 & -2.098458 & -1.461015 \\ \mathrm{H} & 5.063323 & -0.146224 & 1.813428 \\ \mathrm{H} & 4.555270 & 1.044106 & 0.620030 \\ \mathrm{H} & 3.374670 & 0.378876 & 1.754956 \\ \mathrm{H} & -1.374206 & -0.846597 & -2.795344 \\ \mathrm{H} & -1.534299 & -2.176488 & -1.636714 \\ \mathrm{H} & 0.828840 & -3.564164 & -1.649152 \\ \mathrm{H} & 1.486861 & -4.163051 & -0.098386 \\ \mathrm{H} & 0.880122 & -1.136702 & -1.859896 \\ \mathrm{H} & 2.412829 & 0.973340 & -1.126428 \\ \mathrm{H} & 1.355574 & 1.881046 & -2.216232 \\ \mathrm{H} & -1.136122 & 4.500521 & -0.875016 \\ \mathrm{H} & 0.444928 & 4.575242 & -1.692219 \\ \mathrm{H} & -0.600846 & 3.149492 & -1.885772 \\ \mathrm{H} & 1.246161 & 3.797741 & 1.951762 \\ \mathrm{H} & -0.152610 & 4.794883 & 1.483722 \\ \mathrm{H} & & & \\ & & & \end{array}$

Sum of Energies $=-1194.303511$

\section{Pink Pathway (Diels-Alder TS)}

$\begin{array}{lrrr}\text { C } & -2.269460 & -2.632749 & 1.550240 \\ \mathrm{O} & -1.525206 & -2.544271 & 0.345708 \\ \mathrm{C} & -1.167718 & -1.242193 & -0.129372 \\ \mathrm{C} & -0.831834 & -0.208979 & 0.926050 \\ \mathrm{C} & -0.137546 & 0.928351 & 0.493674 \\ \mathrm{C} & 0.914479 & 0.764037 & -0.422032 \\ \mathrm{C} & 1.256213 & -0.584004 & -0.721395 \\ \mathrm{C} & 0.081394 & -1.468737 & -1.012830 \\ \mathrm{C} & 0.933247 & -1.610265 & 1.817921 \\ \mathrm{C} & 1.951513 & -1.144369 & 0.980584\end{array}$




\begin{tabular}{|c|c|c|c|}
\hline $\mathrm{O}$ & 2.478703 & -1.005637 & -1.237313 \\
\hline C & 2.890908 & -1.804268 & -0.065623 \\
\hline $\mathrm{C}$ & 2.668565 & -3.295976 & -0.303792 \\
\hline $\mathrm{C}$ & 4.360795 & -1.500991 & 0.211182 \\
\hline C & -2.281846 & -0.730075 & -1.120121 \\
\hline $\mathrm{C}$ & -3.664821 & -0.562618 & -0.555240 \\
\hline $\mathrm{C}$ & -4.343290 & 0.576725 & -0.335741 \\
\hline $\mathrm{C}$ & -5.762384 & 0.539814 & 0.181449 \\
\hline C & -3.807990 & 1.965073 & -0.586155 \\
\hline $\mathrm{O}$ & 0.096972 & -2.315130 & -1.885151 \\
\hline $\mathrm{C}$ & -0.284852 & 2.286798 & 1.136648 \\
\hline $\mathrm{O}$ & 0.359956 & 3.285299 & 0.344380 \\
\hline $\mathrm{C}$ & 1.691367 & 3.000588 & -0.015878 \\
\hline $\mathrm{O}$ & 1.777478 & 1.752816 & -0.761686 \\
\hline C & 2.622372 & 2.899263 & 1.196019 \\
\hline $\mathrm{C}$ & 2.113404 & 4.086597 & -0.992570 \\
\hline $\mathrm{H}$ & -2.548135 & -3.685851 & 1.646688 \\
\hline $\mathrm{H}$ & 2.410048 & -0.216171 & 1.324527 \\
\hline $\mathrm{H}$ & 3.223435 & -3.606125 & -1.195049 \\
\hline $\mathrm{H}$ & 3.041274 & -3.866276 & 0.554876 \\
\hline $\mathrm{H}$ & 1.619199 & -3.545382 & -0.460314 \\
\hline $\mathrm{H}$ & 4.686752 & -1.994187 & 1.134091 \\
\hline $\mathrm{H}$ & 4.982249 & -1.864688 & -0.614189 \\
\hline $\mathrm{H}$ & 4.519213 & -0.422698 & 0.309429 \\
\hline $\mathrm{H}$ & -1.921860 & 0.196039 & -1.577568 \\
\hline $\mathrm{H}$ & -2.308278 & -1.491483 & -1.909125 \\
\hline $\mathrm{H}$ & -4.188040 & -1.496792 & -0.353459 \\
\hline $\mathrm{H}$ & -5.855051 & 1.090853 & 1.128254 \\
\hline $\mathrm{H}$ & -6.452482 & 1.022468 & -0.525016 \\
\hline $\mathrm{H}$ & -6.111331 & -0.484135 & 0.348255 \\
\hline $\mathrm{H}$ & -4.434757 & 2.494996 & -1.317044 \\
\hline $\mathrm{H}$ & -3.842160 & 2.564829 & 0.334837 \\
\hline $\mathrm{H}$ & -2.779690 & 1.975770 & -0.953678 \\
\hline $\mathrm{H}$ & 0.819869 & -1.182098 & 2.807700 \\
\hline $\mathrm{H}$ & 0.486714 & -2.585173 & 1.659481 \\
\hline $\mathrm{H}$ & -1.674612 & -2.344819 & 2.427521 \\
\hline $\mathrm{H}$ & -3.181526 & -2.025408 & 1.530908 \\
\hline $\mathrm{H}$ & -1.569059 & -0.070229 & 1.714414 \\
\hline $\mathrm{H}$ & -1.332625 & 2.597865 & 1.177505 \\
\hline $\mathrm{H}$ & 0.099731 & 2.295568 & 2.169766 \\
\hline $\mathrm{H}$ & 3.650284 & 2.730925 & 0.863018 \\
\hline $\mathrm{H}$ & 2.582998 & 3.829723 & 1.771430 \\
\hline $\mathrm{H}$ & 2.341318 & 2.072571 & 1.854852 \\
\hline $\mathrm{H}$ & 1.404068 & 4.121687 & -1.823107 \\
\hline $\mathrm{H}$ & 2.122614 & 5.058255 & -0.490581 \\
\hline $\mathrm{H}$ & 3.111526 & 3.873223 & -1.384049 \\
\hline
\end{tabular}

Sum of Energies $=-1194.244081$ 


\section{Green Pathway (Claisen Intermediate)}

\begin{tabular}{|c|c|c|c|}
\hline C & -2.220254 & -2.933066 & -2.336412 \\
\hline $\mathrm{O}$ & -2.197717 & -1.834545 & -1.431618 \\
\hline C & -1.016730 & -1.323109 & -1.005647 \\
\hline $\mathrm{C}$ & 0.254057 & -1.953085 & -1.353087 \\
\hline $\mathrm{C}$ & 1.424258 & -1.384051 & -1.012438 \\
\hline $\mathrm{C}$ & 1.461584 & -0.027303 & -0.372938 \\
\hline $\mathrm{C}$ & 0.168939 & 0.322054 & 0.392737 \\
\hline C & -1.072825 & -0.225605 & -0.177839 \\
\hline $\mathrm{O}$ & -2.264390 & 0.372030 & 0.114822 \\
\hline C & -3.019054 & 0.090853 & 1.338330 \\
\hline $\mathrm{C}$ & -2.931566 & 1.351987 & 2.213451 \\
\hline C & -2.478111 & -1.135542 & 2.084849 \\
\hline $\mathrm{C}$ & -4.461872 & -0.146428 & 0.938904 \\
\hline $\mathrm{C}$ & -4.943494 & -0.197485 & -0.300936 \\
\hline 0 & 0.185672 & 1.091576 & 1.344004 \\
\hline $\mathrm{C}$ & 1.597992 & 1.063336 & -1.515965 \\
\hline $\mathrm{C}$ & 1.757874 & 2.460192 & -0.990813 \\
\hline $\mathrm{C}$ & 0.840300 & 3.440330 & -1.003411 \\
\hline C & -0.557434 & 3.307725 & -1.558753 \\
\hline $\mathrm{C}$ & 1.151967 & 4.787014 & -0.397614 \\
\hline $\mathrm{O}$ & 2.647916 & 0.109089 & 0.405459 \\
\hline C & 3.121472 & -1.056832 & 1.071400 \\
\hline $\mathrm{O}$ & 3.413373 & -2.115189 & 0.132392 \\
\hline $\mathrm{C}$ & 2.779613 & -2.008698 & -1.143994 \\
\hline $\mathrm{C}$ & 2.125397 & -1.585189 & 2.108980 \\
\hline C & 4.447731 & -0.649740 & 1.701134 \\
\hline $\mathrm{H}$ & -3.272065 & -3.076776 & -2.588387 \\
\hline $\mathrm{H}$ & -3.526933 & 1.231674 & 3.126391 \\
\hline $\mathrm{H}$ & -1.890002 & 1.546283 & 2.477555 \\
\hline $\mathrm{H}$ & -3.321244 & 2.212188 & 1.660002 \\
\hline $\mathrm{H}$ & -2.500724 & -2.023552 & 1.446163 \\
\hline $\mathrm{H}$ & -1.456689 & -0.961690 & 2.436751 \\
\hline $\mathrm{H}$ & -3.106557 & -1.333020 & 2.959943 \\
\hline $\mathrm{H}$ & -5.136754 & -0.263148 & 1.788164 \\
\hline $\mathrm{H}$ & -6.002896 & -0.357547 & -0.484347 \\
\hline $\mathrm{H}$ & -4.293881 & -0.083004 & -1.161113 \\
\hline $\mathrm{H}$ & 2.490666 & 0.777754 & -2.086176 \\
\hline $\mathrm{H}$ & 0.734195 & 0.964895 & -2.178051 \\
\hline $\mathrm{H}$ & 2.718105 & 2.661681 & -0.521067 \\
\hline $\mathrm{H}$ & -1.282195 & 3.192355 & -0.741895 \\
\hline $\mathrm{H}$ & -0.841105 & 4.207708 & -2.119373 \\
\hline $\mathrm{H}$ & -0.682982 & 2.447248 & -2.220757 \\
\hline $\mathrm{H}$ & 1.059047 & 5.589465 & -1.143091 \\
\hline $\mathrm{H}$ & 0.441685 & 5.023453 & 0.406705 \\
\hline $\mathrm{H}$ & 2.161986 & 4.823105 & 0.021904 \\
\hline $\mathrm{H}$ & -1.654365 & -2.717466 & -3.250612 \\
\hline
\end{tabular}




$\begin{array}{rrrr}\mathrm{H} & -1.835950 & -3.850325 & -1.873512 \\ \mathrm{H} & 0.243757 & -2.925311 & -1.834982 \\ \mathrm{H} & 3.408754 & -1.407372 & -1.820706 \\ \mathrm{H} & 2.720684 & -3.023236 & -1.550800 \\ \mathrm{H} & 1.207226 & -1.942882 & 1.633864 \\ \mathrm{H} & 2.572187 & -2.429026 & 2.643613 \\ \mathrm{H} & 1.867274 & -0.796593 & 2.821111 \\ \mathrm{H} & 4.286501 & 0.111189 & 2.470254 \\ \mathrm{H} & 4.927255 & -1.522686 & 2.152682 \\ \mathrm{H} & 5.107121 & -0.243190 & 0.929652\end{array}$

Sum of Energies $=-1194.315200$

\section{Green Pathway (Claisen TS)}

$\begin{array}{lrrr}\mathrm{C} & -2.288430 & -3.534019 & -1.440527 \\ \mathrm{O} & -2.284076 & -2.213889 & -0.925450 \\ \mathrm{C} & -1.090269 & -1.635441 & -0.593355 \\ \mathrm{C} & 0.154426 & -2.311471 & -0.714322 \\ \mathrm{C} & 1.330962 & -1.693649 & -0.355032 \\ \mathrm{C} & 1.319726 & -0.341720 & 0.086235 \\ \mathrm{C} & 0.052113 & 0.356992 & 0.333341 \\ \mathrm{C} & -1.148925 & -0.334464 & -0.106261 \\ \mathrm{O} & -2.353448 & 0.314903 & -0.063430 \\ \mathrm{C} & -3.153525 & 0.390008 & 1.157410 \\ \mathrm{C} & -3.037655 & 1.825947 & 1.697228 \\ \mathrm{C} & -2.700421 & -0.628012 & 2.213632 \\ \mathrm{C} & -4.593275 & 0.114745 & 0.769816 \\ \mathrm{C} & -5.030914 & -0.265534 & -0.428369 \\ \mathrm{O} & 0.043710 & 1.517930 & 0.812486 \\ \mathrm{C} & 0.592047 & 2.960264 & -1.141557 \\ \mathrm{C} & 0.955158 & 4.228394 & -0.425585 \\ \mathrm{C} & -0.827784 & 2.875272 & -1.623313 \\ \mathrm{C} & 1.582699 & 2.062681 & -1.499421 \\ \mathrm{C} & 1.371954 & 0.760498 & -1.964019 \\ \mathrm{C} & 2.663317 & -2.388318 & -0.356373 \\ \mathrm{O} & 3.434569 & -1.941692 & 0.759931 \\ \mathrm{C} & 3.641925 & -0.545612 & 0.813178 \\ \mathrm{C} & 4.598327 & -0.061590 & -0.283768 \\ \mathrm{C} & 4.180714 & -0.254733 & 2.208104 \\ \mathrm{O} & 2.402840 & 0.198498 & 0.719838 \\ \mathrm{H} & -3.335165 & -3.766493 & -1.645583 \\ \mathrm{H} & -3.660258 & 1.954716 & 2.590956 \\ \mathrm{H} & -1.995899 & 2.047631 & 1.937114 \\ \mathrm{H} & -3.380570 & 2.534717 & 0.936419 \\ \mathrm{H} & -2.744501 & -1.646014 & 1.816524 \\ \mathrm{H} & -1.682809 & -0.415544 & 2.555906\end{array}$




$\begin{array}{lrrr}\mathrm{H} & -3.367162 & -0.571132 & 3.080983 \\ \mathrm{H} & -5.303581 & 0.271551 & 1.583075 \\ \mathrm{H} & -6.089967 & -0.426815 & -0.613590 \\ \mathrm{H} & -4.342553 & -0.430462 & -1.249243 \\ \mathrm{H} & 0.658198 & 5.112298 & -1.008168 \\ \mathrm{H} & 0.412395 & 4.272593 & 0.526065 \\ \mathrm{H} & 2.026015 & 4.292028 & -0.212425 \\ \mathrm{H} & -1.153040 & 1.859919 & -1.851648 \\ \mathrm{H} & -1.514500 & 3.281839 & -0.876905 \\ \mathrm{H} & -0.932838 & 3.480974 & -2.536980 \\ \mathrm{H} & 2.600224 & 2.319875 & -1.212904 \\ \mathrm{H} & 2.222139 & 0.175919 & -2.297158 \\ \mathrm{H} & 0.413392 & 0.437004 & -2.351924 \\ \mathrm{H} & -1.712864 & -3.610675 & -2.373041 \\ \mathrm{H} & -1.896152 & -4.259035 & -0.714738 \\ \mathrm{H} & 0.182353 & -3.339115 & -1.059966 \\ \mathrm{H} & 2.549841 & -3.467904 & -0.225527 \\ \mathrm{H} & 3.214968 & -2.226731 & -1.296785 \\ \mathrm{H} & 4.758479 & 1.015900 & -0.187143 \\ \mathrm{H} & 5.560831 & -0.573209 & -0.184212 \\ \mathrm{H} & 4.207659 & -0.255416 & -1.286038 \\ \mathrm{H} & 4.302075 & 0.822987 & 2.345415 \\ \mathrm{H} & 5.146762 & -0.748259 & 2.349620 \\ \mathrm{H} & 3.474756 & -0.630557 & 2.952655\end{array}$

Sum of Energies $=-1194.284045$

\section{Green Pathway (Diels-Alder Product)}

$\begin{array}{lrrr}\text { C } & -1.987096 & -3.131537 & 2.421007 \\ \mathrm{O} & -2.316820 & -2.508794 & 1.185116 \\ \mathrm{C} & -1.484276 & -1.530368 & 0.773967 \\ \mathrm{C} & -0.391659 & -1.014338 & 1.364102 \\ \mathrm{C} & 0.248474 & 0.083827 & 0.542200 \\ \mathrm{C} & 0.637215 & -0.523544 & -0.862174 \\ \mathrm{C} & -0.592393 & -1.289163 & -1.457769 \\ \mathrm{C} & -1.802506 & -0.951982 & -0.598532 \\ \mathrm{C} & 3.203201 & -0.766345 & -0.863999 \\ \mathrm{C} & 4.357668 & -1.213771 & -0.345373 \\ \mathrm{C} & 4.519680 & -2.531278 & 0.374831 \\ \mathrm{C} & 5.624848 & -0.400144 & -0.461485 \\ \mathrm{C} & 1.863956 & -1.463428 & -0.877877 \\ \mathrm{O} & -0.558007 & -1.968498 & -2.457947 \\ \mathrm{O} & -3.165733 & -1.063401 & -1.000330 \\ \mathrm{C} & -3.411088 & 0.354850 & -0.619983 \\ \mathrm{C} & -0.867189 & 1.199875 & 0.345960 \\ \mathrm{C} & -1.867529 & 0.589282 & -0.619058\end{array}$




\begin{tabular}{|c|c|c|c|}
\hline $\mathrm{C}$ & -4.122869 & 0.439711 & 0.724772 \\
\hline $\mathrm{C}$ & -4.181259 & 1.058049 & -1.725235 \\
\hline $\mathrm{C}$ & 1.407465 & 0.766576 & 1.269008 \\
\hline 0 & 1.826962 & 1.947543 & 0.585944 \\
\hline $\mathrm{C}$ & 0.816003 & 2.942594 & 0.398340 \\
\hline 0 & -0.331641 & 2.374282 & -0.254836 \\
\hline $\mathrm{C}$ & 1.413116 & 3.956385 & -0.592052 \\
\hline $\mathrm{C}$ & 0.416440 & 3.633938 & 1.714230 \\
\hline $\mathrm{H}$ & -2.746201 & -3.896568 & 2.589373 \\
\hline $\mathrm{H}$ & 0.833778 & 0.302601 & -1.559628 \\
\hline $\mathrm{H}$ & 3.220892 & 0.202641 & -1.363726 \\
\hline $\mathrm{H}$ & 3.594970 & -3.110520 & 0.430950 \\
\hline $\mathrm{H}$ & 5.271415 & -3.155882 & -0.127112 \\
\hline $\mathrm{H}$ & 4.885797 & -2.374542 & 1.399838 \\
\hline $\mathrm{H}$ & 5.450204 & 0.558318 & -0.960150 \\
\hline $\mathrm{H}$ & 6.055486 & -0.193759 & 0.528803 \\
\hline $\mathrm{H}$ & 6.395759 & -0.943182 & -1.026616 \\
\hline $\mathrm{H}$ & 1.773775 & -2.051911 & -1.802002 \\
\hline $\mathrm{H}$ & 1.773332 & -2.179468 & -0.052155 \\
\hline $\mathrm{H}$ & -1.592363 & 0.941843 & -1.618178 \\
\hline $\mathrm{H}$ & -4.263762 & 1.487711 & 1.015305 \\
\hline $\mathrm{H}$ & -5.108444 & -0.032232 & 0.651198 \\
\hline $\mathrm{H}$ & -3.564882 & -0.066429 & 1.516039 \\
\hline $\mathrm{H}$ & -3.677028 & 0.930967 & -2.687635 \\
\hline $\mathrm{H}$ & -5.191250 & 0.641863 & -1.810250 \\
\hline $\mathrm{H}$ & -4.267218 & 2.129857 & -1.510633 \\
\hline $\mathrm{H}$ & -0.993874 & -3.596027 & 2.372136 \\
\hline $\mathrm{H}$ & -2.003272 & -2.405854 & 3.244744 \\
\hline $\mathrm{H}$ & -0.007282 & -1.309691 & 2.334857 \\
\hline $\mathrm{H}$ & -1.297323 & 1.427962 & 1.330624 \\
\hline $\mathrm{H}$ & 1.092846 & 0.995662 & 2.298805 \\
\hline $\mathrm{H}$ & 2.290061 & 0.126353 & 1.325695 \\
\hline $\mathrm{H}$ & 1.986651 & 4.733881 & -0.080144 \\
\hline $\mathrm{H}$ & 2.075719 & 3.436616 & -1.286028 \\
\hline $\mathrm{H}$ & 0.611224 & 4.434213 & -1.160293 \\
\hline $\mathrm{H}$ & -0.047521 & 2.957849 & 2.436168 \\
\hline $\mathrm{H}$ & 1.304598 & 4.069804 & 2.181655 \\
\hline $\mathrm{H}$ & -0.295388 & 4.433726 & 1.495520 \\
\hline
\end{tabular}

Sum of Energies $=-1194.318808$

\section{Green Pathway (Diels-Alder TS)}

$\begin{array}{llll}\mathrm{C} & -1.625803 & 3.591000 & -1.237167 \\ \mathrm{O} & -1.985908 & 2.619868 & -0.266285 \\ \mathrm{C} & -1.213778 & 1.502317 & -0.167614 \\ \mathrm{C} & -0.164150 & 1.174601 & -1.024062\end{array}$




\begin{tabular}{|c|c|c|c|}
\hline $\mathrm{C}$ & 0.381119 & -0.116621 & -0.893338 \\
\hline $\mathrm{C}$ & 0.580760 & -0.585803 & 0.536732 \\
\hline $\mathrm{C}$ & -0.698039 & -0.375000 & 1.401704 \\
\hline $\mathrm{C}$ & -1.676410 & 0.504060 & 0.738837 \\
\hline C & 2.862692 & 0.831676 & 0.622770 \\
\hline $\mathrm{C}$ & 3.206912 & 2.120995 & 0.456959 \\
\hline $\mathrm{C}$ & 2.408387 & 3.315344 & 0.920662 \\
\hline C & 4.506286 & 2.479098 & -0.227713 \\
\hline C & 1.646179 & 0.299764 & 1.327487 \\
\hline $\mathrm{O}$ & -0.832241 & -0.900304 & 2.493110 \\
\hline $\mathrm{O}$ & -3.010241 & 0.506293 & 1.128800 \\
\hline C & -3.529850 & -0.445111 & 0.136207 \\
\hline $\mathrm{C}$ & -2.229496 & -1.183537 & -0.283771 \\
\hline C & -4.219547 & 0.309929 & -0.997581 \\
\hline C & -4.493332 & -1.381926 & 0.859839 \\
\hline $\mathrm{C}$ & 1.433310 & -0.663837 & -1.844781 \\
\hline 0 & 2.400259 & -1.464850 & -1.165315 \\
\hline $\mathrm{C}$ & 1.919970 & -2.468678 & -0.280870 \\
\hline O & 0.860546 & -1.981128 & 0.564670 \\
\hline C & 1.374056 & -3.682389 & -1.040253 \\
\hline $\mathrm{C}$ & 3.112050 & -2.866397 & 0.590522 \\
\hline $\mathrm{H}$ & -2.311368 & 4.426991 & -1.088991 \\
\hline $\mathrm{H}$ & 3.544772 & 0.085908 & 0.226333 \\
\hline $\mathrm{H}$ & 1.440238 & 3.056402 & 1.353938 \\
\hline $\mathrm{H}$ & 2.970677 & 3.888586 & 1.671327 \\
\hline $\mathrm{H}$ & 2.226246 & 4.003902 & 0.083332 \\
\hline $\mathrm{H}$ & 5.058767 & 1.590114 & -0.547354 \\
\hline $\mathrm{H}$ & 4.329858 & 3.108304 & -1.112139 \\
\hline $\mathrm{H}$ & 5.157331 & 3.062233 & 0.439216 \\
\hline $\mathrm{H}$ & 1.929894 & -0.346932 & 2.167442 \\
\hline $\mathrm{H}$ & 1.098630 & 1.132931 & 1.773629 \\
\hline $\mathrm{H}$ & -2.003276 & -1.995761 & 0.404398 \\
\hline $\mathrm{H}$ & -4.643720 & -0.395338 & -1.721497 \\
\hline $\mathrm{H}$ & -5.029486 & 0.922414 & -0.589183 \\
\hline $\mathrm{H}$ & -3.529216 & 0.972873 & -1.524093 \\
\hline $\mathrm{H}$ & -3.990012 & -1.856429 & 1.707363 \\
\hline $\mathrm{H}$ & -5.353256 & -0.820227 & 1.240211 \\
\hline $\mathrm{H}$ & -4.859441 & -2.159726 & 0.179606 \\
\hline $\mathrm{H}$ & -0.592953 & 3.934697 & -1.095846 \\
\hline $\mathrm{H}$ & -1.740419 & 3.205792 & -2.259657 \\
\hline $\mathrm{H}$ & 0.055783 & 1.783097 & -1.895771 \\
\hline $\mathrm{H}$ & 0.970174 & -1.231852 & -2.664607 \\
\hline $\mathrm{H}$ & 2.013859 & 0.148565 & -2.294022 \\
\hline $\mathrm{H}$ & 0.935903 & -4.394687 & -0.335300 \\
\hline $\mathrm{H}$ & 0.600819 & -3.395162 & -1.756881 \\
\hline $\mathrm{H}$ & 2.183137 & -4.175975 & -1.587507 \\
\hline $\mathrm{H}$ & 2.804651 & -3.644427 & 1.295038 \\
\hline $\mathrm{H}$ & 3.927521 & -3.249335 & -0.031147 \\
\hline
\end{tabular}




$\begin{array}{lrrr}\mathrm{H} & 3.476965 & -2.008378 & 1.158893 \\ \mathrm{C} & -1.482292 & -1.218163 & -1.462747 \\ \mathrm{H} & -0.980658 & -2.144177 & -1.717157 \\ \mathrm{H} & -1.744888 & -0.591367 & -2.310341\end{array}$

Sum of Energies $=-1194.240586$ 